\title{
Parathyroid hormone-dependent bone formation requires butyrate production by intestinal microbiota
}

\author{
Jau-Yi Li, 1,2 Mingcan Yu, ${ }^{1,2}$ Subhashis Pal, ${ }^{1,2}$ Abdul Malik Tyagi, ${ }^{1,2}$ Hamid Dar, ${ }^{1,2}$ Jonathan Adams, ${ }^{1,2}$ M. Neale Weitzmann, ${ }^{1,2,3}$ \\ Rheinallt M. Jones, ${ }^{2,4,5}$ and Roberto Pacifici ${ }^{1,2,5}$ \\ ${ }^{1}$ Division of Endocrinology, Metabolism and Lipids, Department of Medicine, and ${ }^{2}$ Emory Microbiome Center, Emory University, Atlanta, Ceorgia, USA. ${ }^{3}$ Atlanta Department of Veterans Affairs Medical Center, \\ Decatur, Georgia, USA. ${ }^{4}$ Division of Pediatric Gastroenterology, Hepatology, and Nutrition, Department of Pediatrics, and ${ }^{5}$ Immunology and Molecular Pathogenesis Program, Emory University, Atlanta, \\ Georgia, USA.
}

\begin{abstract}
Parathyroid hormone (PTH) is a critical regulator of skeletal development that promotes both bone formation and bone resorption. Using microbiota depletion by wide-spectrum antibiotics and germ-free (GF) female mice, we showed that the microbiota was required for PTH to stimulate bone formation and increase bone mass. Microbiota depletion lowered butyrate levels, a metabolite responsible for gut-bone communication, while reestablishment of physiologic levels of butyrate restored PTH-induced anabolism. The permissive activity of butyrate was mediated by GPR43 signaling in dendritic cells and by GPR43-independent signaling in T cells. Butyrate was required for PTH to increase the number of bone marrow (BM) regulatory T cells (Tregs). Tregs stimulated production of the osteogenic Wnt ligand Wnt10b by BM CD8+ T cells, which activated Wnt-dependent bone formation. Together, these data highlight the role that butyrate produced by gut luminal microbiota plays in triggering regulatory pathways, which are critical for the anabolic action of PTH in bone.
\end{abstract}

\section{Introduction}

Parathyroid hormone (PTH) is a calciotrophic hormone produced by the parathyroid glands. PTH is a critical regulator of skeletal development and postnatal skeletal maturation due to its capacity to stimulate both bone formation and bone resorption (1). Accordingly, lack of PTH leads to decreased trabecular bone in fetal and neonatal mice (2). PTH may induce bone loss or promote bone mass acquisition, mainly depending on whether target cells are exposed to PTH continuously or intermittently. The bone catabolic activity of PTH is most pronounced when the hormone is produced or infused in excessive amounts and in a continuous fashion (3), as it happens in primary and secondary hyperparathyroidism (4). In these conditions, PTH stimulates bone resorption and, to a lesser extent, bone formation, leading to net bone loss ( 5 , 6). Although stimulation of bone formation is a constant response elicited by PTH, the bone anabolic activity of PTH is maximized by daily injections of PTH in young mice, a treatment modality known as intermittent PTH (iPTH) treatment. This intervention markedly increases bone volume and strength due to a stimulation of bone formation tempered by a more moderate increase in resorption $(6,7)$. Stimulation of bone formation is due to increased osteoblast formation and lifespan, and reactivation of bone lining cells. These effects of iPTH are secondary to activation of Wnt signaling in osteoblastic cells $(7,8)$.

Related Commentary: https://doi.org/10.1172/JCI135712

Conflict of interest: The authors have declared that no conflict of interest exists. Copyright: @ 2020, American Society for Clinical Investigation.

Submitted: September 16, 2019; Accepted: December 23, 2019; Published: March 3, 2020. Reference information: / Clin Invest. 2020;130(4):1767-1781.

https://doi.org/10.1172/JCl133473.
In the mouse, iPTH activates Wnt signaling through multiple mechanisms, including blunted osteocytic production of the Wnt inhibitor sclerostin (9-11) and decreased production by osteoblasts of the Wnt inhibitor Dkk1 (12). These effects result from direct activation of the $\mathrm{PTH} / \mathrm{PTHrP}$ receptor in osteoblasts and osteocytes $(13,14)$. In addition, reports in humans and animals show that teriparatide activates Wnt signaling by inducing the production of Wnt10b, an osteogenic Wnt ligand produced by T cells (15-18). Animal studies further show that the relevant pool of Wnt10b derives from BM CD8 ${ }^{+} \mathrm{T}$ cells (1618). Accordingly, global or T cell-specific silencing of Wnt10b production blocks the capacity of iPTH to increase bone formation and trabecular bone volume (16-18). By contrast, the anabolic effects of iPTH in cortical bone are completely $\mathrm{T}$ cell independent (16-18), likely due to the fact that $\mathrm{T}$ cells have no contact with periosteal surfaces and have limited capacity to communicate with osteocytes.

iPTH causes an approximately 2 - to 3 -fold increase in the number of Tregs in humans and mice, which is required for iPTH to exert its bone anabolic activity (19). Tregs are suppressive helper $\mathrm{CD}^{+} \mathrm{T}$ cells that play an essential role in dampening inflammation and maintaining immune tolerance (20). Reports have highlighted the bone-regulating capacities of Tregs, describing mechanisms where Tregs blunt bone resorption $(21,22)$, stimulate bone formation by promoting the differentiation of osteoblasts (23), and are pivotal for the stimulation of bone formation induced by nutritional supplementation with the probiotic Lactobacillus rhamnosus GG (LGG) $(24,25)$.

The enzymatic activity harbored within some taxa of gut microbiota can digest carbohydrates to generate millimolar concentrations of the short-chain fatty acids (SCFAs) butyrate, propionate, and acetate (26). SCFAs selectively support the development 
of peripheral Tregs (27-29). This suggests that microbiota-induced generation of SCFAs in the gut may promote Treg differentiation in BM, which is pivotal for the bone anabolic activity of iPTH.

Here we examined the role of the microbiota and of butyrate in the regulation of bone responses to iPTH in young female mice. We report that the activity of $\mathrm{iPTH}$ is blocked in mice lacking or depleted of the microbiota, or the SCFA receptor GPR43, and that in microbiota-depleted mice the activity of $\mathrm{PTTH}$ is reestablished by restoration of permissive amounts of circulating butyrate.

\section{Results}

iPTH treatment does not induce bone anabolism in germ-free mice and antibiotic-treated conventional mice. To investigate the role of the microbiota in the bone anabolic activity of PTH, C57BL6 female mice were raised in either conventional (Conv.R) or germfree (GF) conditions and treated with vehicle or iPTH for 4 weeks, starting at the age of 8 weeks. Analysis by in vitro $\mu \mathrm{CT}$ of femurs harvested at 12 weeks of age revealed that iPTH increased trabecular bone volume fraction (BV/TV) and trabecular thickness (Tb.Th) in Conv.R mice but not in GF mice (Figure 1, A-C). Trabecular number (Tb.N) and trabecular separation (Tb.Sp), which are additional indices of trabecular structure, were altered by iPTH in Conv.R controls but not in GF mice (Supplemental Figure 1, A and B; supplemental material available online with this article; https://doi.org/10.1172/JCI133473DS1). To confirm the relevance of the microbiota for the bone anabolic activity of $\mathrm{PTH}$, in a second experiment Conv.R mice were treated with vehicle or iPTH for 4 weeks starting at the age of 8 weeks, and either with or without broad-spectrum antibiotics $(1 \mathrm{mg} / \mathrm{mL}$ ampicillin, 0.5 $\mathrm{mg} / \mathrm{mL}$ vancomycin, $1 \mathrm{mg} / \mathrm{mL}$ neomycin sulfate, $1 \mathrm{mg} / \mathrm{mL}$ metronidazole, dissolved in water) for 6 weeks, starting at the age of 6 weeks. Treatment with antibiotics decreased gut bacterial load by more than $99 \%$ (Supplemental Figure 2). iPTH increased BV/ $\mathrm{TV}$ and Tb.Th in control Conv.R mice but not in those treated with antibiotics (Figure 1, D-F). Similarly, iPTH increased Tb.N and decreased Tb.Sp in control Conv.R mice, while it was ineffective in antibiotic-treated mice (Supplemental Figure 1, C and D). Analysis of cortical structure by $\mu \mathrm{CT}$ revealed that $\mathrm{PTH}$ increased cortical area (Ct.Ar) and cortical thickness (Ct.Th) in control Conv.R mice as well as GF mice, and in Conv.R antibiotic-treated mice (Figure 1, G-J). Together, these findings indicate that the microbiome is required for the anabolic activity of iPTH in trabecular bone, but it is not implicated in the mechanism whereby iPTH increases cortical bone mass.

To investigate the role of the microbiome in the skeletal response to iPTH of older mice, Conv.R mice were treated with vehicle or iPTH and either with or without antibiotics for 4 weeks, starting at 6 months of age. Control mice treated with vehicle had a lower BV/TV than the corresponding group of antibiotic-treated mice, reflecting the life-long proresorptive effect of the microbiota $(30,31)$. Confirming a requirement for the microbiota, iPTH increased $\mathrm{BV} / \mathrm{TV}$ and $\mathrm{Tb}$.Th in control mice but not in mice treated with antibiotics (Figure 1, K and L). As a result, control mice and antibiotic-treated mice treated with $\mathrm{PPTH}$ had a similar BV/TV ratio. Similarly, iPTH increased Tb.N and decreased Tb.Sp in control mice, while it was ineffective in antibiotic-treated mice (Supplemental Figure 1, E and F).
Analysis of femurs harvested at 12 weeks of age by histomorphometry revealed that iPTH increased the dynamic indices of bone formation mineral apposition rate (MAR) and bone formation rate $(\mathrm{BFR} / \mathrm{BS})$ in the trabecular compartment of Conv.R mice, but not of GF mice (Figure 2, A-C). Similarly, iPTH increased trabecular MAR and BFR/BS in control Conv.R mice, but not in Conv.R mice treated with antibiotics (Figure 2, D-F). Analysis of static indices of bone formation revealed similar findings, as iPTH failed to increase the number of osteoblasts per bone surface (N.Ob/BS) and the percentage of surfaces covered by osteoblasts (Ob.S/BS) in the trabecular bone of GF mice and Conv.R mice treated with antibiotics, while it increased these indices in microbiota-replete mice (Figure 2, G-J). Moreover, iPTH induced a significant increase in the 2 indices of trabecular bone resorption, osteoclast per bone surface (N.Oc/BS) and percentage of surfaces covered by osteoclasts (Oc.S/BS), in control mice but not in GF mice and Conv.R mice treated with antibiotics (Figure $2, \mathrm{~K}-\mathrm{P}$ ). These data indicate that the microbiota is required for iPTH to increase formation and resorption in cancellous bone. By contrast, iPTH increase cortical MAR and cortical BFR/BS in control and antibiotic-treated Conv.R mice, confirming that the microbiota is not required for the anabolic activity of iPTH in cortical bone (Figure 2, Q and R).

Measurements of serum levels of osteocalcin, a marker for bone formation, revealed that iPTH increased osteocalcin levels in all groups of 12-week-old mice, although to a lesser degree in mice lacking the microbiome (Supplemental Figure 3, A and B). Serum CTX, a marker for bone resorption, also increased significantly in response to iPTH in all groups of mice (Supplemental Figure 3, C and D). These findings are in agreement with the fact that osteocalcin and CTX reflect primarily cortical bone turnover.

To determine the role of the microbiome on osteoblastogenesis, BM was harvested at 12 weeks of age and cultured for 1 week, and stromal cells (SCs) were purified and used to determine their rate of proliferation and apoptosis as previously described $(16,18$, 19). These experiments revealed that iPTH failed to increase the proliferation of SCs from GF mice or Conv.R mice treated with antibiotics, while it stimulated SC proliferation in microbiotareplete groups (Figure 3, A and B). Moreover, iPTH had no effects on the rate of SC apoptosis in GF mice or antibiotic-treated Conv.R mice, while it decreased SC apoptosis in control Conv.R mice (Figure 3, C and D). Analysis of the expression levels of osteoblast differentiation genes in SCs revealed that iPTH treatment increased the expression of type 1 collagen, Runx2, osterix, bone sialoprotein, and osteocalcin mRNAs in SCs from microbiotareplete mice, while it had no effect on SCs from mice raised in GF conditions and in SCs from antibiotic-treated Conv.R mice (Figure 3, $\mathrm{E}$ and $\mathrm{F}$ ). These findings demonstrate that $\mathrm{iPTH}$ regulates osteoblast proliferation, differentiation, and life span through a microbiome-dependent mechanism.

Because osteoblast proliferation and differentiation are induced by activation of canonical Wnt signaling (32), we measured transcript enrichment of genes upregulated in response to Wnt signaling. Transcript levels of 9 measured Wnt-responsive genes were increased by iPTH in SCs of control mice, but not in SCs from GF mice or from antibiotic-treated Conv.R mice (Figure 3, $\mathrm{G}$ and $\mathrm{H}$ ), indicating that iPTH activated Wnt signaling in SCs through a microbiota-dependent mechanism. 
A
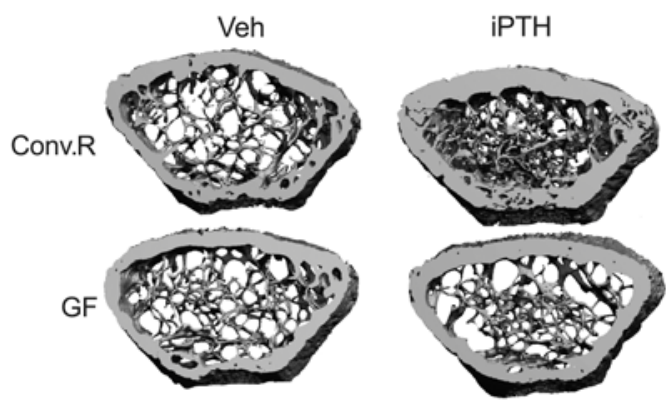

D

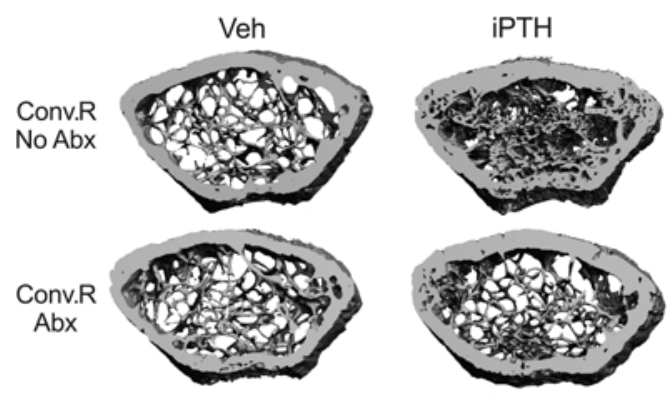

G

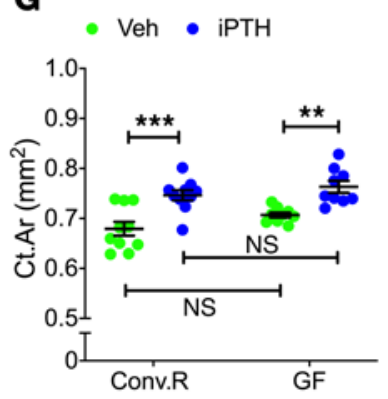

H
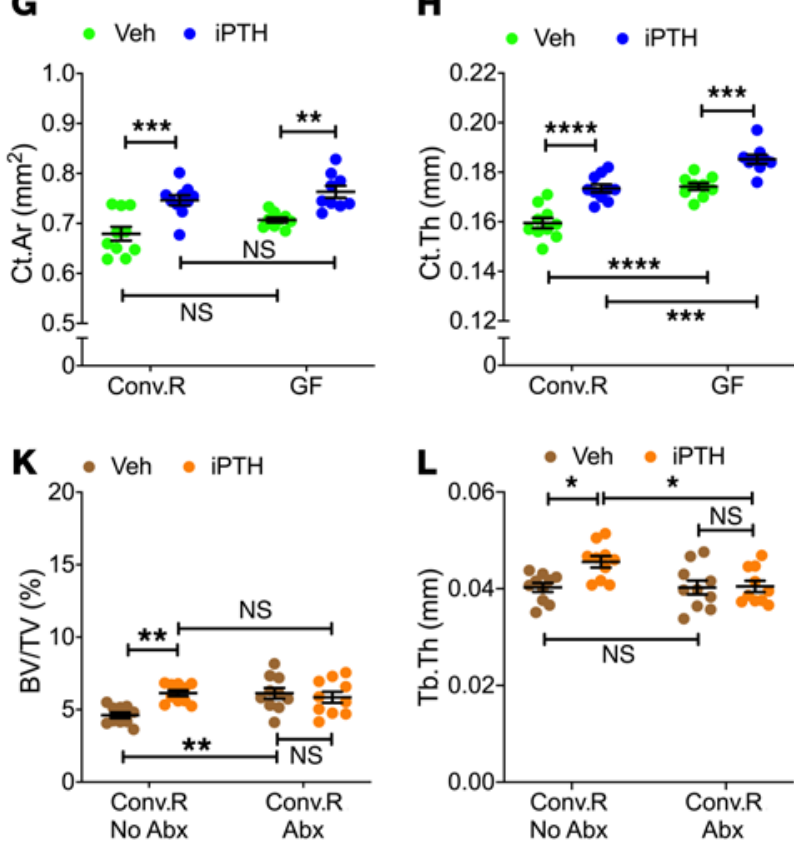

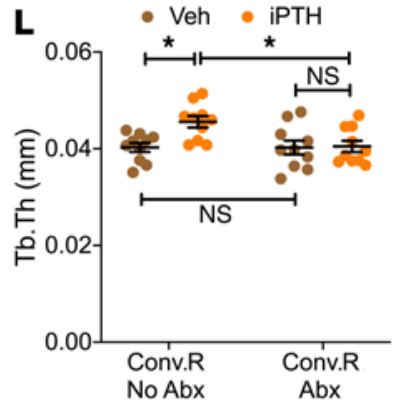

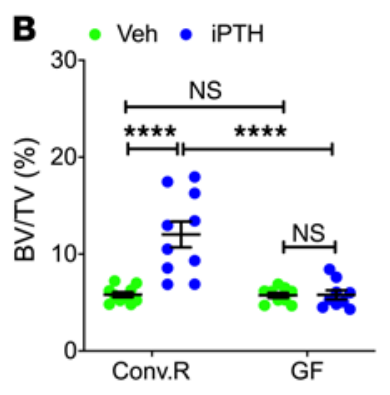
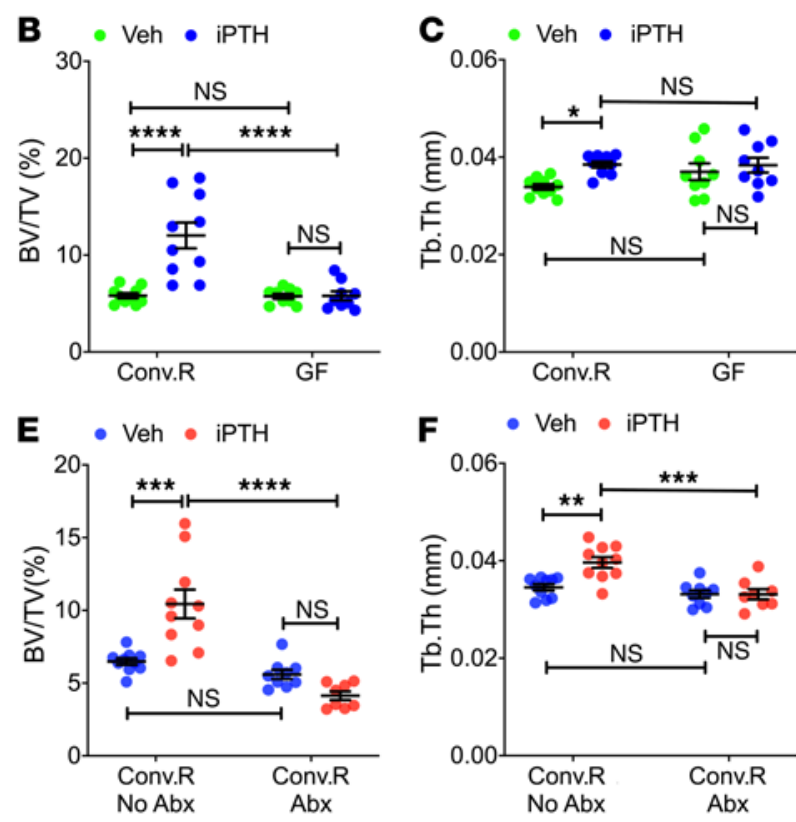

I

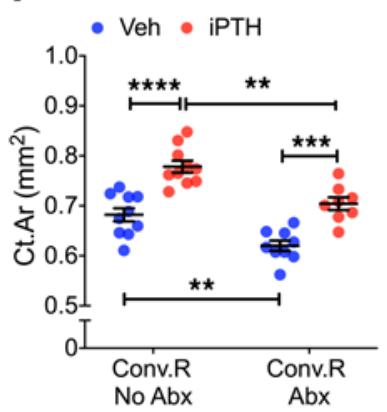

$\mathbf{J}$

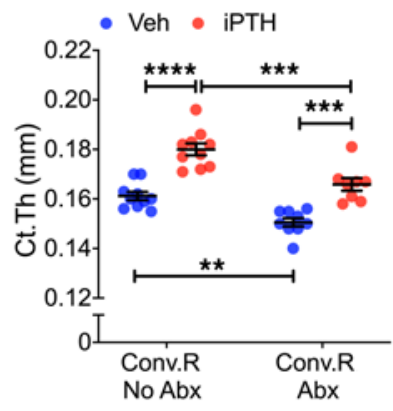

Figure 1. IPTH treatment fails to improve trabecular bone structure in GF mice and conventional mice treated with antibiotics. (A) Images of representative 3D $\mu \mathrm{CT}$ reconstructions of examined femurs from 12-week-old conventionally raised (Conv.R) mice and GF mice. (B and C) $\mu \mathrm{CT}$ scanning measurements of trabecular bone volume fraction (BV/TV) and trabecular thickness (Tb.Th) in 12-week-old Conv.R mice and GF mice ( $n=9-10$ mice/group). (D) Images of representative $3 \mathrm{D} \mu \mathrm{CT}$ reconstructions of examined femurs from 12-week-old Conv.R mice treated with and without antibiotics (Abx). (E and $\mathbf{F}$ ) $\mu \mathrm{CT}$ scanning measurements of BV/TV and Tb.Th in 12-week-old Conv.R mice treated with and without Abx ( $n=8-10$ mice/group).(G and $\mathbf{H}) \mu C T$ scanning measurements of cortical bone area (Ct.Ar), and cortical thickness (Ct.Th) in 12-week-old Conv.R mice and GF mice ( $n=9$-10 mice/group). (I and J) $\mu$ CT scanning measurements of Ct.Ar and Ct.Th in 12-week-old Conv.R mice treated with and without Abx ( $n=8-10$ mice/group). (K and $\mathbf{L}) \mu C T$ scanning measurements of BV/TV and Tb.Th in 7-month-old Conv.R mice treated with and without Abx ( $n=10$ mice/group). Data were expressed as mean \pm SEM. All data were normally distributed according to the Shapiro-Wilk normality test. All data were analyzed by 2-way ANOVA and post hoc tests, applying Bonferroni's correction for multiple comparisons. ${ }^{*} P<0.05,{ }^{* *} P<0.01,{ }^{* *} P<0.001$, and ${ }^{* * * *} P<0.0001$ compared with the indicated group in the post hoc tests.

Previous reports demonstrate that $\mathrm{CD}^{+} \mathrm{T}$ cell-produced Wnt10b is critical for the anabolic activity of $\mathrm{iPTH}$ in the trabecular compartment of the skeleton (16-18). Therefore, we investigated whether the microbiome is required for $\mathrm{iPTH}$ to upregulate Wnt10b expression. iPTH increased Wnt10b transcripts in whole $\mathrm{BM}$ cells and $\mathrm{BM} \mathrm{CD}^{+} \mathrm{T}$ cells from microbiota-replete mice, but not in those from GF mice and antibiotic-treated Conv.R mice (Figure 3, I-L).

iPTH increases the number of BM Tregs in humans and mice (19). This effect is critical for the bone anabolic activity of iPTH, 
A

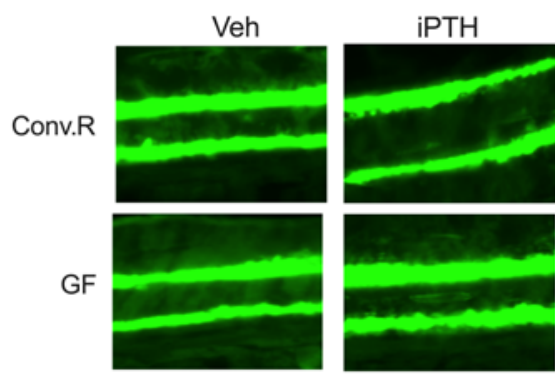

D
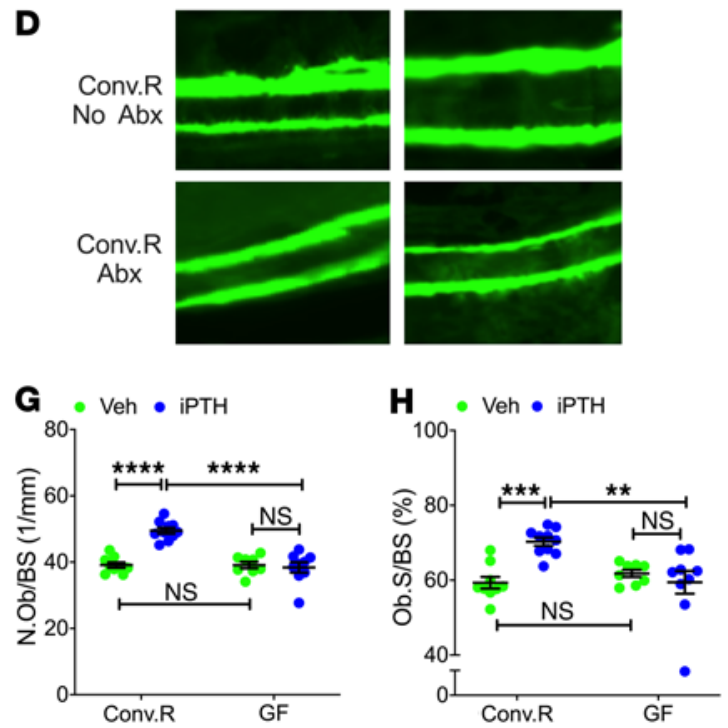

$\mathbf{K}$

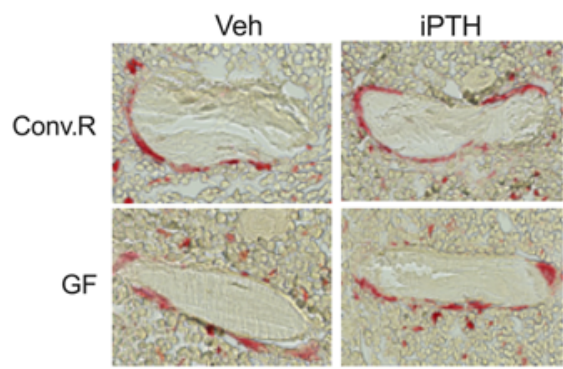

$\mathbf{N}$
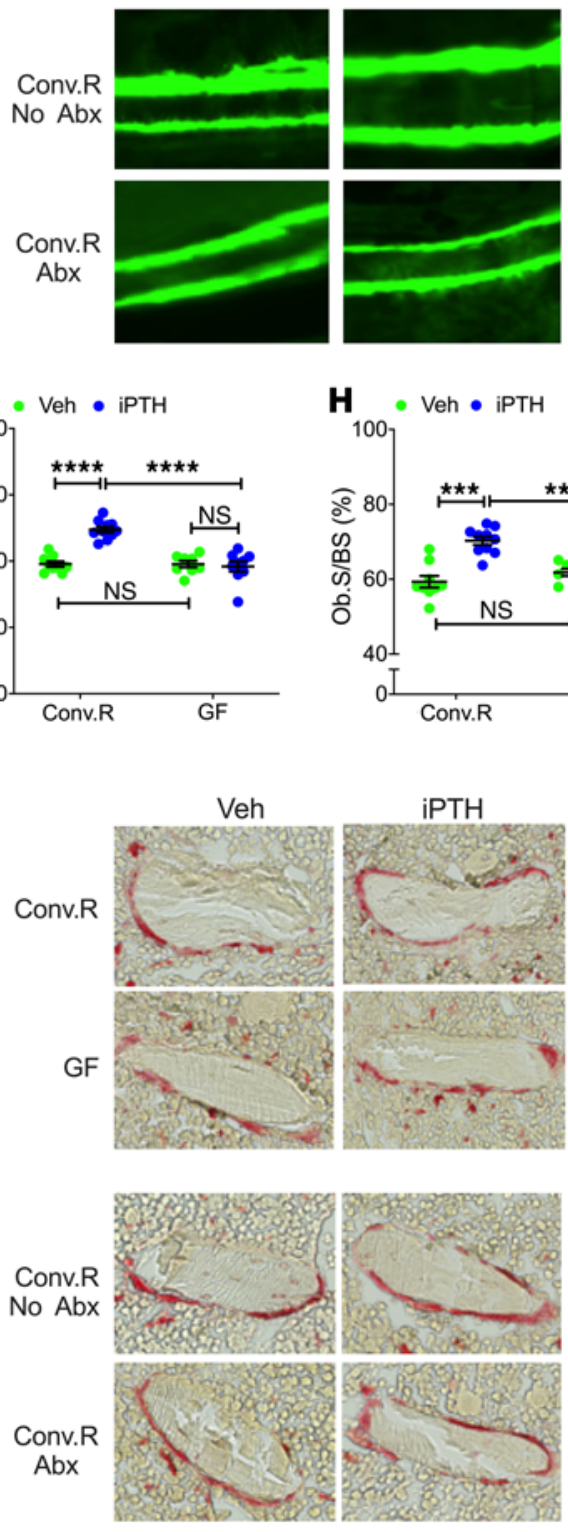
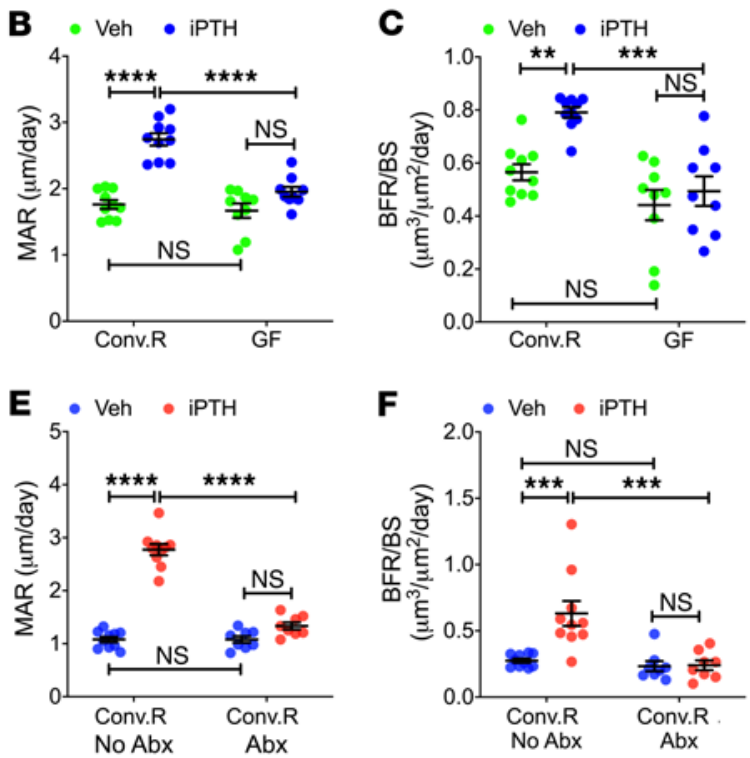

F $\bullet$ Veh $-\mathrm{iPTH}$

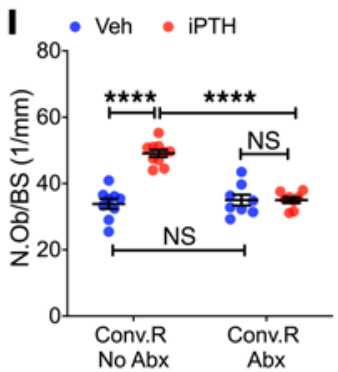

J
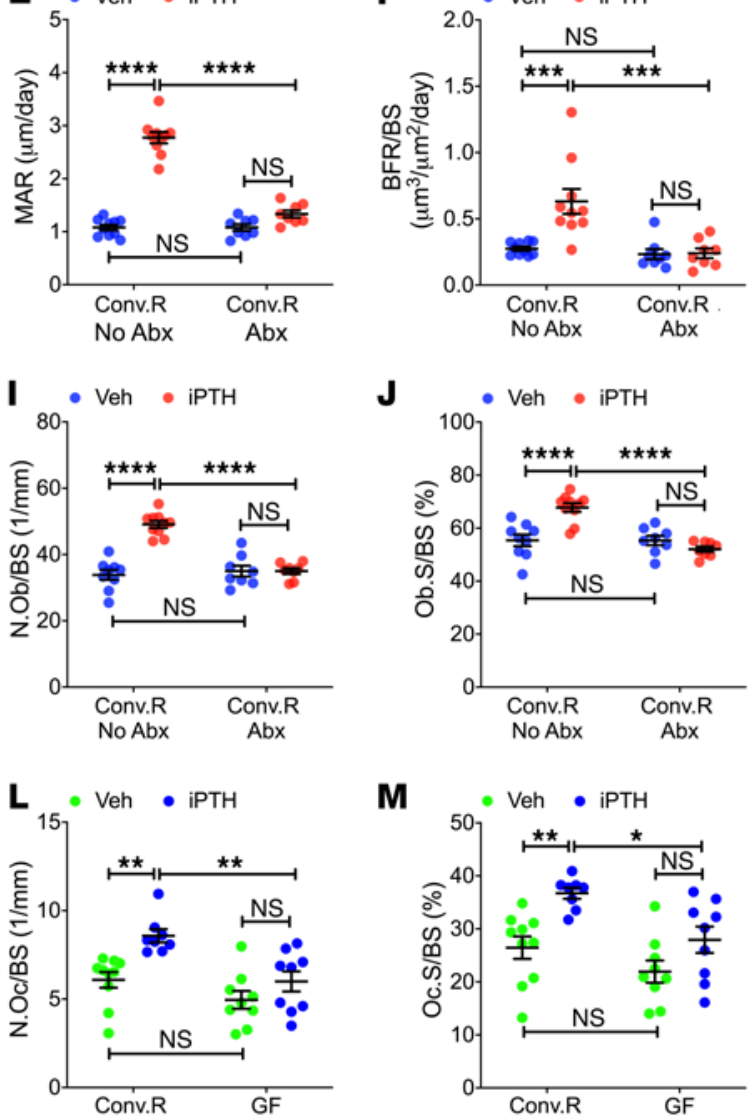

M
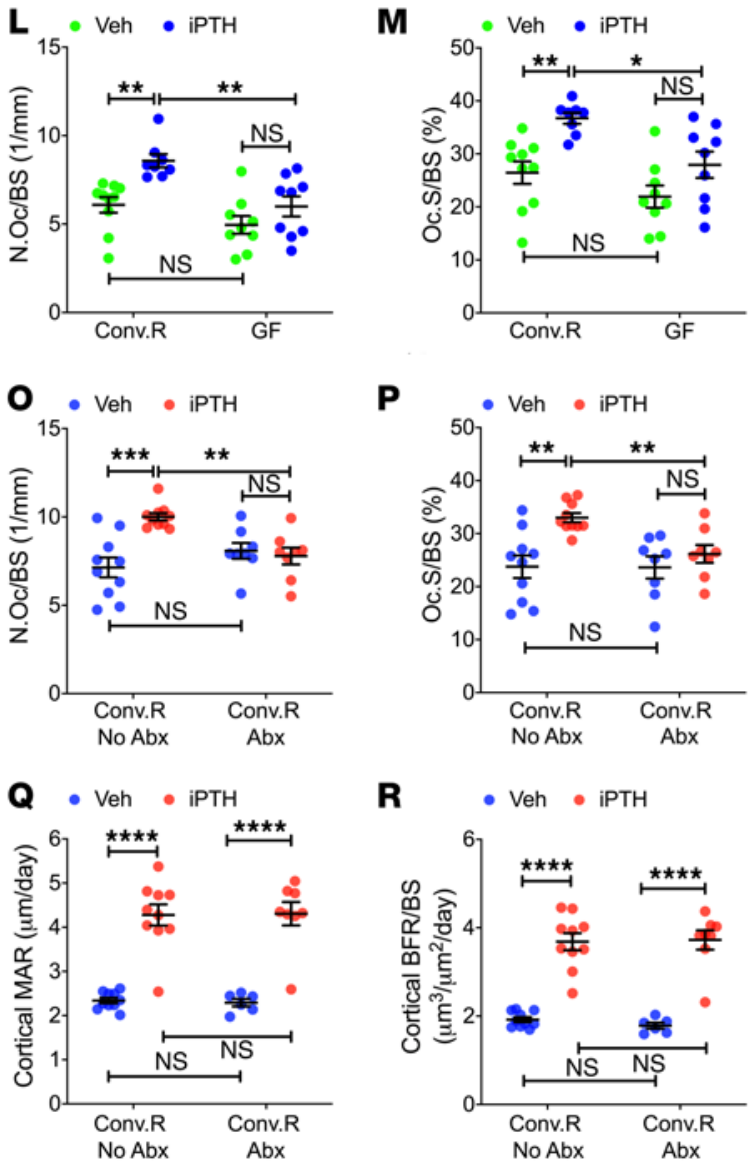

R $\bullet$ Veh $\bullet$ iPTH

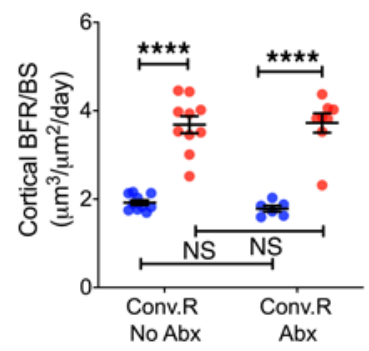


Figure 2. iPTH treatment fails to stimulate trabecular bone turnover in 12-week-old GF mice and conventional mice treated with antibiotics. Mice were treated with iPTH or vehicle for 4 weeks. Mice were sacrificed and analyzed at 12 weeks of age. (A) Images are representative sections from Conv.R mice and GF mice displaying trabecular calcein doublefluorescence labeling. Original magnification $\times 20$. (B and C) Trabecular MAR and trabecular bone formation rate per $\mathrm{mm}$ bone surface (BFR/BS) in Conv.R mice and GF mice ( $n=9-10$ mice/group). (D) Images are representative sections from Conv.R mice treated with and without antibiotics (Abx), displaying trabecular calcein double-fluorescence labeling. Original magnification $\times 20$. ( $\mathbf{E}$ and $\mathbf{F}$ ) Trabecular MAR and BFR/BS in Conv.R mice treated with and without $A b x$ ( $n=8-10$ mice/group). ( $\mathbf{G}$ and $\mathbf{H})$ The number of osteoblasts per mm bone surface (N.Ob/BS) and the percentage of bone surface covered by osteoblasts (Ob.S/BS) in trabecular bone from Conv.R mice and GF mice ( $n=9-10$ mice/group). (I and J) N.Ob/BS and Ob.S/BS in trabecular bone from Conv.R mice treated with and without $A b x$ ( $n=8-10$ mice/group). (K and $\mathbf{N}$ ) The images show tartrate-resistant acid phosphatase-stained (TRAP-stained) sections of the distal femur. Original magnification $\times 40$. ( $\mathbf{L}$ and $\mathbf{M}$ ) The number of osteoclasts per $\mathrm{mm}$ bone surface (N.Oc/BS) and the percentage of bone surface covered by osteoclasts (Oc.S/BS) in trabecular bone from Conv.R mice and GF mice ( $n=$ 9-10 mice/group). (O and P) N.Oc/BS and Oc.S/BS in trabecular bone from Conv.R mice treated with and without $A b x$ ( $n=8-10$ mice/group). ( $Q$ and R) Cortical MAR and BFR/BS in Conv.R mice treated with and without Abx ( $n=8-10$ mice/group). Data are expressed as mean \pm SEM. All data were normally distributed according to the Shapiro-Wilk normality test and analyzed by 2-way ANOVA and post hoc tests, applying Bonferroni's correction for multiple comparisons. ${ }^{*} P<0.05$, ${ }^{* *} P<0.01$, ${ }^{* *} P<0.001$, and ${ }^{* * * *} P<0.0001$ compared with the indicated group in the post hoc tests.

as an increase in the number of Tregs is required for iPTH to induce production of Wnt10b by BM CD8 ${ }^{+} \mathrm{T}$ cells (19). The microbiota controls the induction and maintenance of colonic Tregs via multiple mechanisms (33), suggesting that the microbiota might not only be required for the generation of intestinal Tregs in unstimulated conditions, but also for iPTH to expand Tregs. We used flow cytometry to test this hypothesis (Supplemental Figure 4). Since the calculation of the absolute number of Peyer's patches (PP) Tregs is technically challenging due to variability of the size of the collected PP tissue, PP Tregs were quantified only as percentage of total $\mathrm{CD} 4^{+} \mathrm{T}$ cells. We found that $\mathrm{PTH}$ treatment increased the number of PP Tregs and BM Tregs in microbiota-replete mice, but not in GF mice and antibiotic-treated Conv.R mice (Figure 3, $\mathrm{M}-\mathrm{P})$. Analysis of vehicle-treated groups revealed that GF mice and antibiotic-treated mice had a lower number of PP and BM Tregs, as compared with Conv.R mice not treated with antibiotics (Figure 3, M-P).

TGF- $\beta 1$ and IGF- 1 are factors capable of promoting Tregs differentiation (33-35) induced by iPTH (36-38). In this study, we assessed the role of the microbiome on the capacity of iPTH to stimulate the production of TGF- $\beta 1$ and IGF- 1 in the BM. iPTH treatment increased Tgfbl and Igfl transcripts in the BM of microbiota-replete Conv.R mice but not in the BM of GF mice and Conv.R mice treated with antibiotics (Figure 3, Q-T). These findings indicate that the microbiota is required for $\mathrm{PTH}$ to increase the production of TGF- $\beta 1$ and IGF- 1 in the BM.

Gut microbiota production of butyrate enables $i$ PTH to promote bone anabolism. Butyrate and other SCFAs, produced in the murine cecum and human colon by bacterial fermentation of nondigestible material (39), play a central role in the differentiation of colonic Tregs through actions on dendritic cells (DCs) and naive CD4 ${ }^{+} \mathrm{T}$ cells (27-29).

We investigated the hypothesis that the microbiota produces permissive levels of SCFAs that are required for iPTH to expand Tregs and induce bone anabolism. Serum levels of butyrate were lower in antibiotic-treated Conv.R mice than in mice not treated with antibiotics (Figure 4A), indicating that the microbiota contributed to the generation of circulating butyrate. In antibiotictreated mice, nutritional supplementation with butyrate $(5 \mathrm{mM})$ increased serum butyrate to levels similar to those measured in mice not treated with antibiotics (Figure 4A). Moreover, butyrate supplementation restored the capacity of iPTH to increase BV/TV and $\mathrm{Tb}$.Th in antibiotic-treated mice (Figure 4, B and C). In antibiotic-treated mice, butyrate supplementation also restored the capacity of $\mathrm{PPTH}$ to maximally increase serum osteocalcin and CTX levels (Figure 4, D and E), expand PP and BM Tregs (Figure 4, F-H), increase Wnt10b expression by $\mathrm{CD}^{+} \mathrm{T}$ cells (Figure 4I), and increase BM Tgfbl and Igfl mRNA levels (Figure 4, J and $\mathrm{K})$. These findings indicate that microbiota-produced butyrate is necessary for iPTH to promote bone anabolism.

Both butyrate and iPTH facilitates Treg differentiation, raising the question of the identity of the cellular targets of butyrate and iPTH relevant for Treg differentiation. Butyrate dose dependently increased the number of Tregs in cultures of $\mathrm{CD} 4^{+} \mathrm{T}$ cells stimulated by anti-CD3 and anti-CD28 antibody-coated beads, IL-2, and TGF- $\beta$ (Figure 5A). Propionate and acetate did not increase Treg differentiation in cultures of WT naive $\mathrm{CD} 4^{+} \mathrm{T}$ cells, whereas a mixture of all 3 SCFAs was as effective as butyrate alone (Figure $5 \mathrm{~B}$ ). These findings confirmed that butyrate has the capacity to directly target $\mathrm{CD} 4^{+} \mathrm{T}$ cells (27). In vitro butyrate treatment increased the transcript levels of the Treg-inducing factors $T g f b 1$ and Igfl in cultures of WT BM cells (Figure 5, C and D), indicating that butyrate may also potentiate Treg differentiation indirectly. By contrast, PTH failed to increase Treg differentiation when added to cultures of WT naive $\mathrm{CD} 4^{+} \mathrm{T}$ cells (Figure $5 \mathrm{E}$ ), indicating that PTH did not directly target $\mathrm{CD} 4^{+} \mathrm{T}$ cells.

We also found an increase in Treg differentiation in anti-CD3 antibody and TGF- $\beta$-stimulated cocultures of DCs pretreated in vitro with butyrate for 6 hours and untreated $\mathrm{CD} 4^{+} \mathrm{T}$ cells (Figure $5 F)$. By contrast, pretreatment of DCs with PTH was ineffective (Figure 5G). These findings are consistent with the notion that targeting of DCs is one of the mechanisms by which butyrate induces Treg differentiation (27). The data also indicate that PTH did not directly target DCs.

To further investigate cellular targets of butyrate and PTH relevant for Treg differentiation, 8-week-old Conv.R mice were treated with antibiotics for 4 weeks to dampen butyrate levels. These mice were also treated with vehicle, iPTH, or butyrate for the last 2 weeks of the experiment. DCs and naive $\mathrm{CD} 4^{+} \mathrm{T}$ cells were sorted and cocultured in the presence of anti-CD3 antibody and TGF- $\beta$. In this ex vivo assay, cocultures of DCs from butyratetreated mice and $\mathrm{CD} 4^{+} \mathrm{T}$ cells from either iPTH-treated or butyrate-treated mice were the most efficient in generating Tregs (Figure 5H). A smaller yet significant increase in Treg differentiation was also detected in all the other groups except cocultures of DCs from iPTH-treated mice and T cells from vehicle-treated mice. These data suggested that butyrate affects both $\mathrm{T}$ cells and 
A

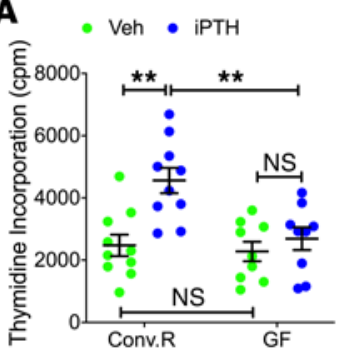

E

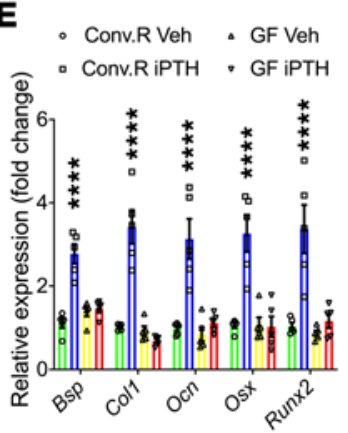

I
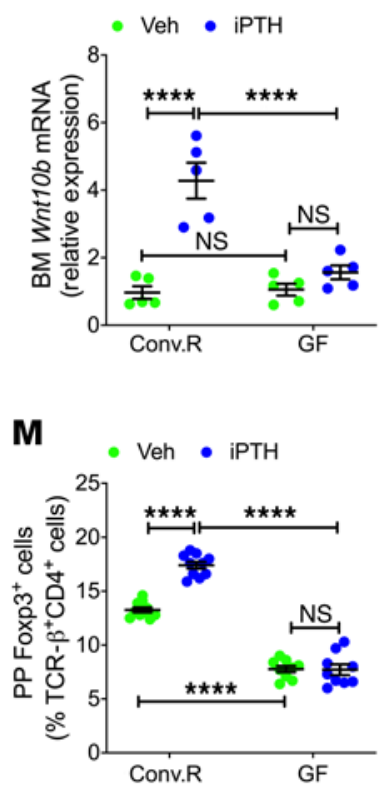

$\mathbf{Q}$

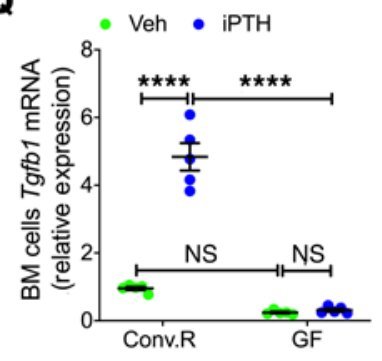

B
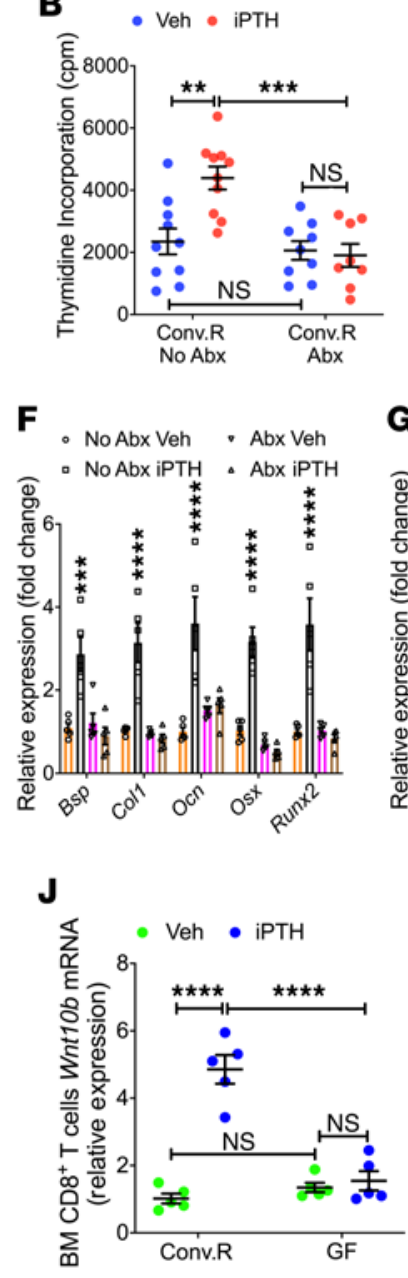

$\mathbf{N}$

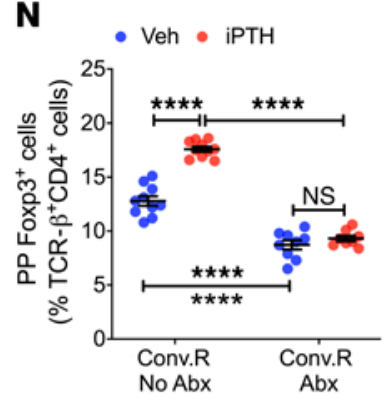

$\mathbf{R}$

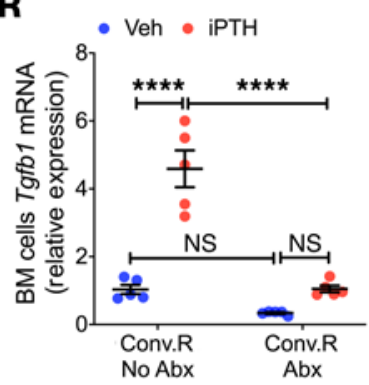

C $\vee$ Veh $\bullet$ PTH
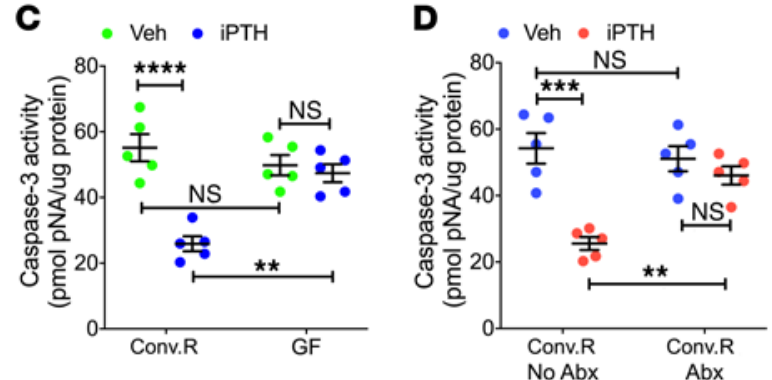

G C Conv.R Veh : GF Veh

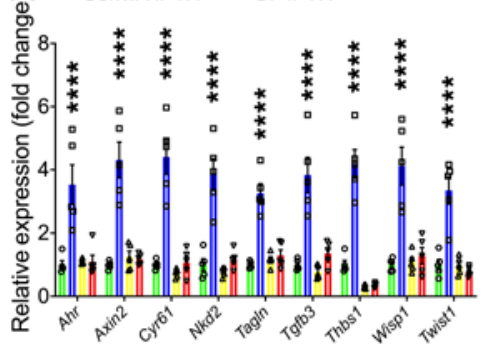

H.NoAbx Veh \& Abx Veh

- No Abx IPTH - Abx IPTH

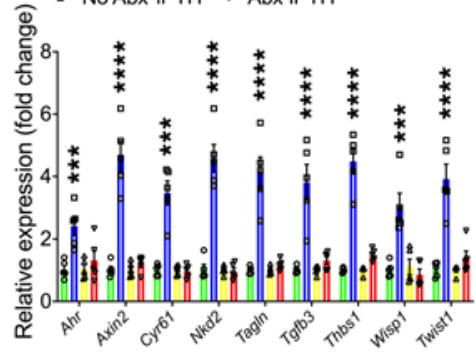

$\mathbf{K}$

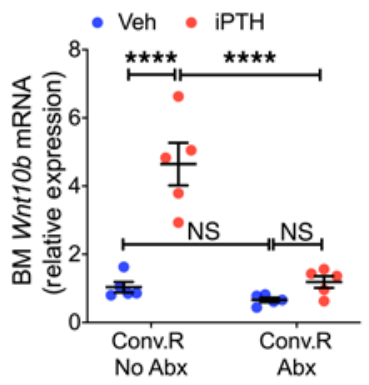

0

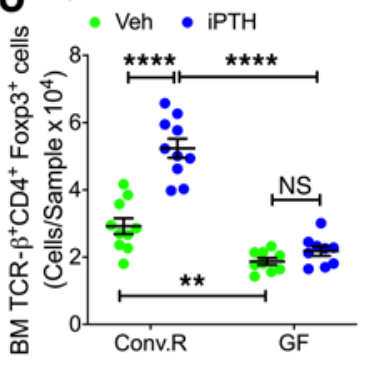

S

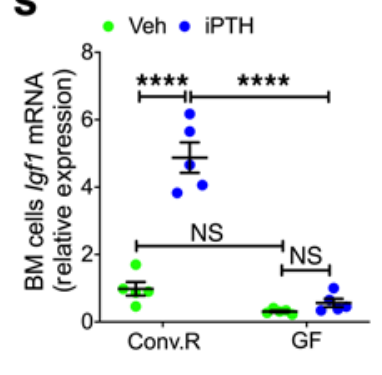

$\mathbf{L}$
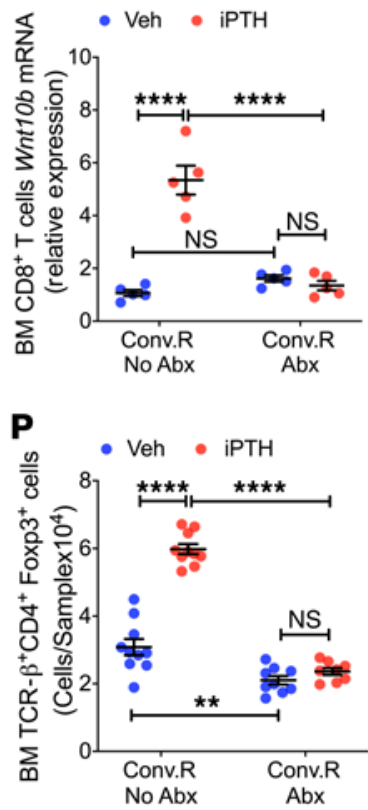

$\mathbf{T}$

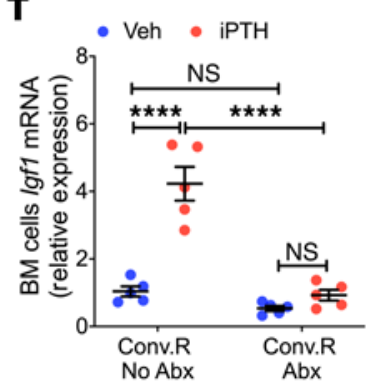


Figure 3. iPTH treatment fails to regulate SC proliferation and life span, $T$ cell expression of Wnt10b, number of Tregs, and BM production of TGF- $\boldsymbol{\beta}$ and IGF-1. (A and B) SC proliferation as measured by thymidine incorporation ( $n=8-10$ mice/group). (C and D) SC apoptosis as measured by Caspase-3 activity ( $n=5$ mice/group). (E and $\mathbf{F}$ ) mRNA levels of bone sialoprotein (Bsp), type 1 collagen (Col1), osteocalcin (Ocn), osterix (Osx), and Runx2, which are factors representative of the differentiation of SCs into osteoblasts ( $n=5$ mice/group). ( $\mathbf{G}$ and $\mathbf{H}$ ) Transcripts of genes that are specifically increased by Wnt signaling in SCs. The analyzed genes were aryl-hydrocarbon receptor (Ahr), Axin2, cysteine rich protein 61 (Cyr61), naked cuticle 2 homolog ( $N k d 2$ ), transgelin (Tagln), transforming growth factor $\beta 3$ (Tgfb3), thrombospondin 1 (Thbs 1 ), Wnt1 inducible signaling pathway protein 1 (Wisp1), and Twist gene homolog 1 (Twist1) ( $n=5$ mice/ group). (I-L) Wnt10b mRNA levels in whole BM cells and sorted BM CD8 ${ }^{+}$ T cells ( $n=5$ mice/group). (M-P) PP and BM Tregs (TCR- $\beta^{+}$CD4 $4^{+}$Foxp $3^{+}$cells) ( $n=8-10$ mice/group). (Q and R) BM Tgfb1mRNA levels ( $n=5$ mice/group). (S and T) BM Igf- 1 mRNA levels ( $n=5$ mice/group). Data were expressed as mean \pm SEM. All data were normally distributed according to the Shapiro-Wilk normality test. All data were analyzed by 2-way ANOVA and post hoc tests, applying Bonferroni's correction for multiple comparisons. ${ }^{* *} P<$ $0.01,{ }^{* *} P<0.001$, and ${ }^{* * * *} P<0.0001$ compared with the indicated group in the post hoc tests.

DCs whereas iPTH regulates T cells but not DCs. Because in vitro treatment with PTH did not stimulate Treg differentiation (Figure $5 E)$, iPTH is likely to affect T cells indirectly. Since the effects of butyrate on DCs and CD4 ${ }^{+} \mathrm{T}$ cells were only partially additive, the signaling events activated by butyrate in these 2 cell lineages are likely to converge on a common downstream target.

iPTH expands Tregs through a GPR43-mediated mechanism. Butyrate induces Treg differentiation primarily via $\mathrm{T}$ cell intrinsic epigenetic regulation of the Foxp3 gene (28). An additional means of Treg induction by SCFAs is the activation of the cell surface signaling receptor GPR $43(29,40)$. To investigate the specific mechanisms involved, 8-week-old female GPR $43^{-/-}$mice and WT littermates were treated with iPTH for 4 weeks. Analysis by in vitro $\mu \mathrm{CT}$ of femurs harvested at sacrifice revealed that iPTH increased BV/ $\mathrm{TV}$ and improved indices of trabecular structure in $\mathrm{GPR} 43^{+/+}$mice but not in GPR43 ${ }^{-/}$mice (Figure 6, A and B; and Supplemental Figure 5, A and B). In GPR43-/- mice, iPTH also failed to increase serum osteocalcin and CTX levels (Figure 6, C and D), expand PP and BM Tregs (Figure 6, E-G), and increase Wnt10b expression by whole BM cells and $\mathrm{CD}^{+} \mathrm{T}$ cells (Figure $6, \mathrm{H}$ and I). These findings indicated that the bone anabolic activity of iPTH occurs via GPR43-mediated signaling.

To provide additional evidence that butyrate mediates the bone anabolic effect of iPTH through GPR43 signaling, GPR $43^{-/-}$mice and WT littermates were treated with antibiotics and $\mathrm{PPTH}$ and supplemented with $5 \mathrm{mM}$ butyrate. Butyrate supplementation restored the capacity of iPTH to increase BV/TV, indices of trabecular structure, and serum markers of bone turnover in WT mice but not in GPR43-/- (Figure 6, J-L; and Supplemental Figure 5, C-E), confirming the functional relevance of GPR43 for butyrate signaling.

GPR43 signaling in T cells and/or DCs could be necessary for iPTH to expand Tregs. To investigate the role of GPR43 signaling in T cells, TCR- $\beta^{-/-}$mice, a strain completely devoid of $\alpha \beta \mathrm{T}$ cells, were reconstituted with sorted splenic T cells from GPR $43^{-/-}$ mice or GPR $43^{+/+}$littermates. Two weeks after adoptive transfer, which is a period sufficient for engraftment and expansion of $\mathrm{T}$ cells (24), all mice were treated with either vehicle or iPTH for 4 weeks. iPTH increased BV/TV and Tb.Th, as well as osteocalcin and CTX levels, in mice reconstituted with GPR $43^{-/-} \mathrm{T}$ cells and in those reconstituted with GPR $43^{+/+} \mathrm{T}$ cells (Figure 7, A-D). In addition, iPTH expanded PP and BM Tregs (Figure 7, E and F), and increased $\mathrm{BM}$ and $\mathrm{CD} 8^{+} \mathrm{T}$ cells levels of Wnt10b transcripts in all mice (Figure 7, G and $\mathrm{H}$ ). These findings indicated that GPR43 signaling in T cells was not required for iPTH to induce bone anabolism. This hypothesis was confirmed by the finding that in vitro treatment with butyrate dose-dependently increased the formation of Tregs in cultures of GPR $43^{-/-}$and GPR $43^{+/+}$naive $\mathrm{CD} 4^{+} \mathrm{T}$ cells (Figure 7I).

Since GPR43 expression is restricted to myeloid cells among hematopoietic cells (41), and considering that butyrate potentiates the capacity of DCs to induce Treg differentiation (27), butyrate may regulate DCs by activating GPR43 signaling. To investigate the relevance of GPR43 signaling in DCs, experiments were conducted using cocultures of DCs from GPR $43^{-/-}$or GPR $43^{+/+}$mice pretreated with butyrate for 6 hours and untreated GPR $43^{+/+}$naive $\mathrm{CD}^{+}{ }^{+} \mathrm{T}$ cells. These cocultures were stimulated by anti-CD3 antibody and TGF- $\beta$. We found that butyrate pretreatment of DCs increased Treg differentiation in cocultures containing GPR $43^{+/+}$ DCs, but not in those containing GPR $43^{-/-}$DCs (Figure 7J).

To investigate the role of GPR43 signaling in DCs in an ex vivo model, DCs and naive $\mathrm{CD} 4^{+} \mathrm{T}$ cells were sorted from antibiotic-treated GPR $43^{-/-}$mice and WT littermates at the end of a 2-week-long treatment period with vehicle or butyrate. DCs and $\mathrm{CD}^{+} \mathrm{T}$ cells were then cocultured in the presence of anti-CD3 antibody and TGF- $\beta$. The highest number of Tregs was yielded by cocultures of T cells and DCs from WT mice treated with butyrate, and by cocultures of GPR $43^{-/-}$T cells and WT DCs, both obtained from mice treated with butyrate (Figure $7 \mathrm{~K}$ ). However, cocultures with GPR43 $3^{--}$DCs and WT T cells, both from butyrate-treated mice yielded a lower number of Tregs. Cocultures with both $\mathrm{T}$ cells and DCs from butyrate-treated GPR $43^{-/-}$mice yielded similar number of Tregs as cocultures of GPR $43^{-/-}$DC cells and WT $\mathrm{T}$ cells from butyrate-treated mice (Figure $7 \mathrm{~K}$ ), confirming that butyrate targets DCs via GPR43, whereas it regulates CD4 ${ }^{+} \mathrm{T}$ cells via GPR43-independent mechanisms.

\section{Discussion}

We reported that the production of permissive amounts of butyrate from the gut microbiota was required for iPTH to induce bone anabolism in young female mice. Microbial production of butyrate potentiated the capacity of iPTH to induce the differentiation of naive $\mathrm{CD} 4^{+} \mathrm{T}$ cells into Tregs, a population of $\mathrm{T}$ cells that induces conventional $\mathrm{CD}^{+} \mathrm{T}$ cells to release Wnt10b. This osteogenic Wnt ligand activates Wnt signaling in osteoblastic cells and stimulates bone formation (16-18). Butyrate enables iPTH to expand Tregs via GPR43 signaling in DCs and GPR43independent targeting of $\mathrm{T}$ cells. Whether this mechanism is sex dependent remains to be determined.

Tregs are a population of predominantly $\mathrm{CD} 4^{+} \mathrm{T}$ cells that play a critical role in maintaining immune tolerance and immune homeostasis (20). Tregs are also recognized as important bone regulators. Tregs reside preferentially on the endosteal surfaces of bone (42), where they regulate osteoclast formation $(21,22,43)$, blunt bone 
A

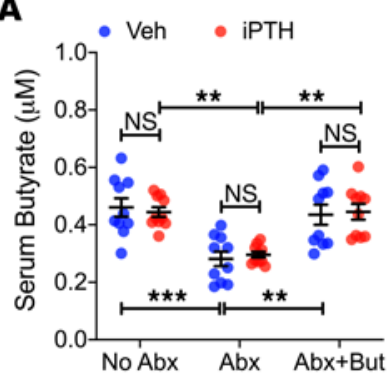

D

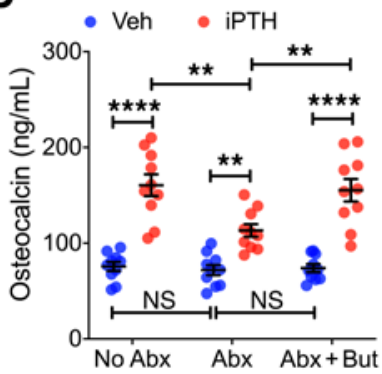

$\mathbf{F}$

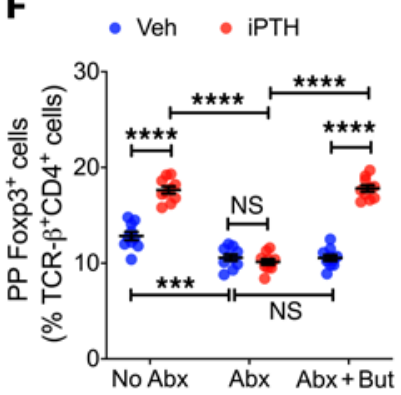

I

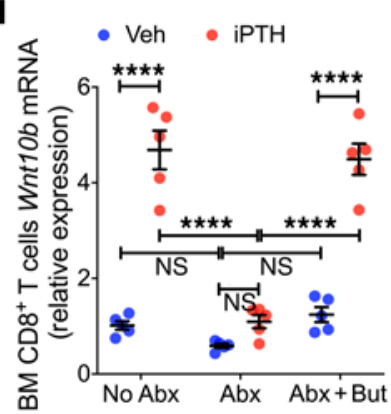

B

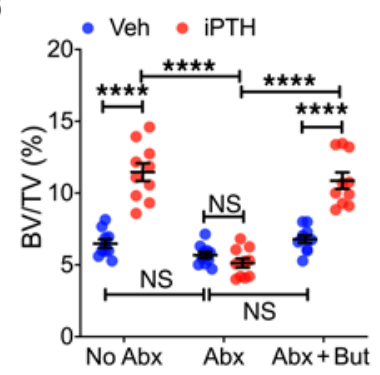

E

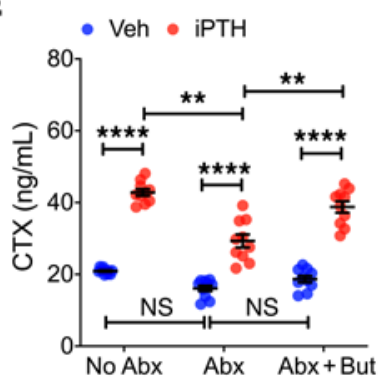

G

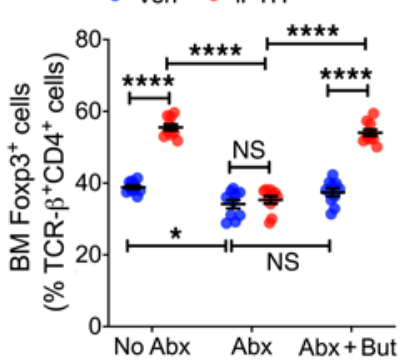

J

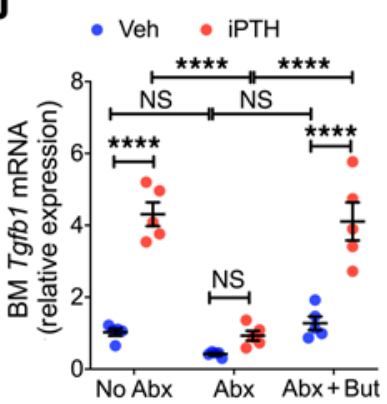

C

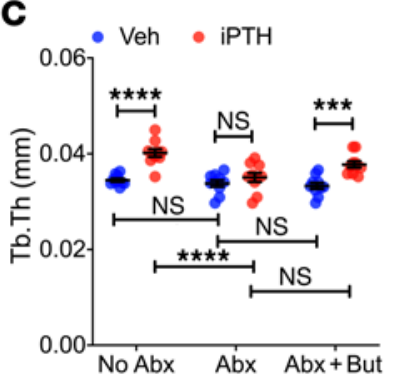

Figure 4. Butyrate supplementation restores the capacity of iPTH to induce bone anabolism, stimulate bone turnover, expand Tregs, induce Wn10b expression, and upregulate the levels of Tgf $\beta 1$ and Igf1 in microbiota-depleted mice. Mice treated with vehicle or $\mathrm{PPTH}$, and butyrate (But) for 4 weeks, starting at 8 weeks of age. Mice were also treated with antibiotics (Abx) for 6 weeks, starting at 6 weeks of age. Mice were sacrificed and analyzed at 12 weeks of age.

(A) Serum butyrate concentrations ( $n=10$ mice/group). (B and C) Bone volume fraction (BV/TV) and trabecular thickness (Tb.Th) ( $n=10$ mice/ group). (D and E) Serum osteocalcin and CTX levels ( $n=10$ mice/group). (F-H) PP and BM Tregs (TCR- $\beta^{+} \mathrm{C}-$ D4 ${ }^{+}{ }{ }{ }^{2} 3^{+}$cells) ( $n=10$ mice/group). (I) BM CD8+ T cell Wnt10b mRNA levels ( $n=5$ mice/group). (J) BM Tgfb1 mRNA levels ( $n=5$ mice/group). (K) BM Igf- 1 mRNA levels ( $n=5$ mice/ group). Data were expressed as mean \pm SEM. All data were normally distributed according to the Shapiro-Wilk normality test. All data were analyzed by 2-way ANOVA and post hoc tests, applying Bonferroni's correction for multiple comparisons. ${ }^{*} P<0.05$, ${ }^{*} P$ $<0.01$, ${ }^{* *} P<0.001$, and ${ }^{* * * *} P<$ 0.0001 compared with the indicated group in the post hoc tests. resorption $(21,44)$, and prevent ovariectomy-induced bone loss (45). In addition, Tregs are recognized as mediators of the bone anabolic activity of the probiotic LGG $(24,25)$ and of iPTH (19). Supporting a role for Tregs in the skeletal activity of iPTH are reports that blockade of Treg expansion silences the bone anabolic activity of PTH (19).

The intestinal microbiota controls the induction and maintenance of intestinal Tregs via multiple mechanisms, including costimulation of naive $\mathrm{CD} 4^{+} \mathrm{T}$ cells by $\mathrm{DCs}$ activated by bacterial antigens (46), signals from Toll-like receptors (TLRs) and other microbial pattern recognition molecules (47), IL-2 produced by $\mathrm{T}$ cells activated by bacterial antigens (48), TGF- $\beta$, IGF-1, retinoic acid, and a number of additional microbiota-derived, host cellderived, and diet-derived factors (33). In addition, the intestinal microbiota promotes Treg differentiation in distant organs via

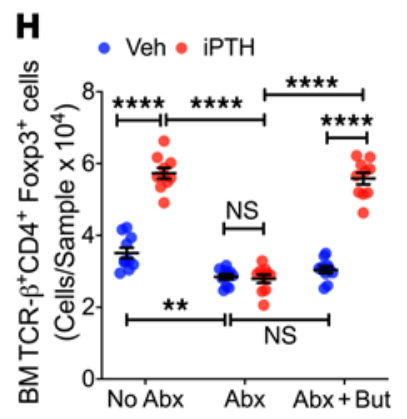

K

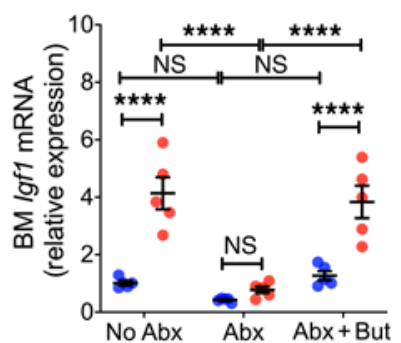

SCFAs derived from bacterial fermentation of complex nondigestible carbohydrates present in the diet $(33,49)$.

In the current study, we found antibiotic treatment to decrease the circulating levels of butyrate by approximately $50 \%$ and to block the expansion of intestinal and BM Tregs induced by iPTH. The capacity of iPTH to expand Tregs was reestablished by supplementation with a dose of butyrate, which restored physiologic circulating levels of butyrate. Based on these findings, we concluded that permissive levels of butyrate were required for iPTH to regulate Treg differentiation.

The mechanism by which SCFAs expand Tregs has been extensively investigated (27-29). SCFAs induce the differentiation of naive $\mathrm{CD} 4^{+} \mathrm{T}$ cells into Tregs through a receptorindependent mechanism that causes acetylation of histone $\mathrm{H} 3$ 
A

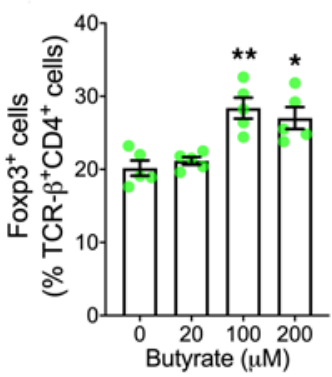

E

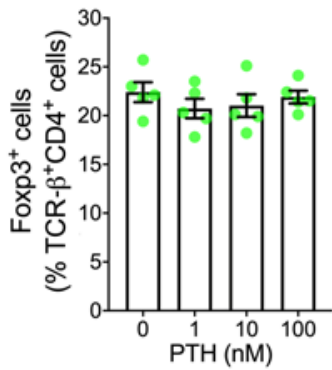

B

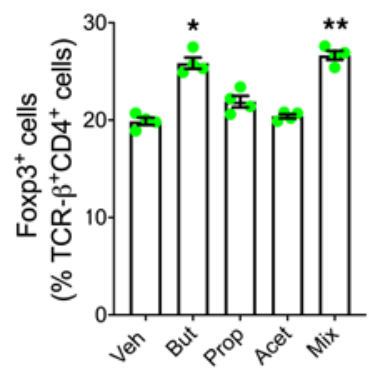

$\mathbf{F}$

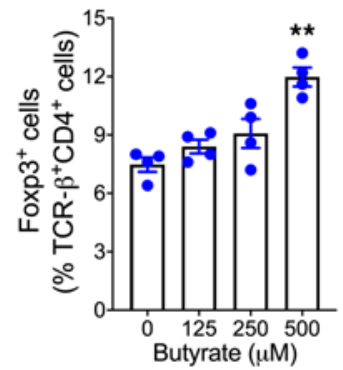

C

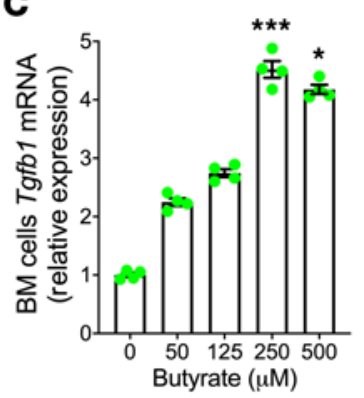

G

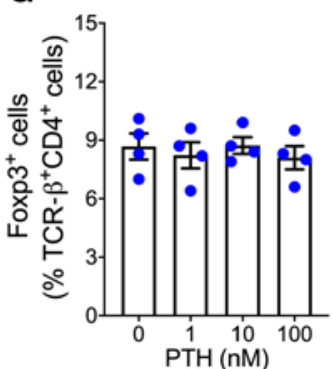

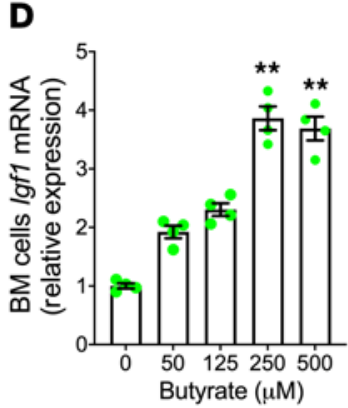

H

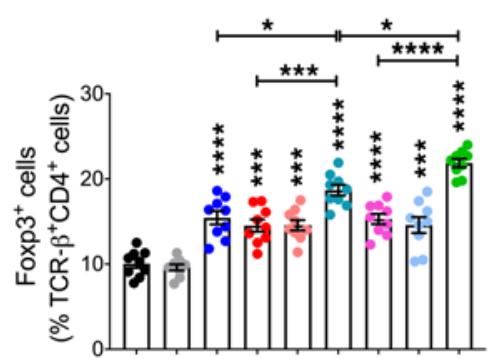

CD4+ $\mathrm{T}$ cells: Veh-treated mice +++ + - - - - -

CD4+ $\mathrm{T}$ cells: iPTH-treated mice - - - - - - + + + +

CD4+ $\mathrm{T}$ cells: But treated mice - - - + + + - - -

DCs: Veh-treated mice + - - + - - + - -

DCs: iPTH-treated mice - + - - + - - + -

DCs: But-treated mice - - + + - + + + - +
Figure 5. Butyrate, but not PTH, directly stimulates Treg differentiation. (A) Effect of butyrate on the number of Tregs in cultures of naive $C D 4^{+}$T cells ( $n=5 /$ group). Figure shows 1 representative experiment of 3 experiments. (B) Effect of SCFAs ( $100 \mu \mathrm{M}$ each) on the number of Tregs in cultures of naive CD4 ${ }^{+}$T cells ( $n=4 /$ group). (C and D) Effect of butyrate on Tgf $\beta 1$ and Igf- 1 mRNA levels in cultures of BM cells ( $n=4$ /group). Figure shows 1 representative experiment of 3 experiments. (E) Effect of PTH on the number of Tregs in cultures of naive CD4+ $4^{+}$cells ( $n=5$ /group). (F and $\mathbf{G}$ ) Number of Tregs in cocultures of untreated naive CD4+ $T$ cells and DCs pretreated in vitro for 6 hours with butyrate or PTH ( $n=4$ /group). Figure shows 1 representative experiment of 3 experiments. (H) Number of Tregs in cocultures of naive CD4+ $\mathrm{T}$ cells and DCs. In this experiment, 8-weekold Conv.R mice were treated with antibiotics for 4 weeks and iPTH or butyrate during the last 2 weeks. DCs and naive CD4+ ${ }^{+}$cells were sorted and cocultured ( $n=9 /$ group, from 2 separate experiments). In A, B, and $\mathbf{E}$, the cultures were stimulated by anti-CD3 and anti-CD28 antibodies, IL-2, and TCF- $\beta$. In F-H, the cultures were stimulated by anti-CD3 antibody and TGF- $\beta$. Data were expressed as mean \pm SEM. In A-G, data were analyzed by Kruskal-Wallis and Dunn's multiple comparisons nonparametric tests, as they were not normally distributed as assessed by Shapiro-Wilk normality test. In $\mathbf{H}$, data were normally distributed according to the Shapiro-Wilk normality test and analyzed by 1-way ANOVA and post hoc tests, applying Bonferroni's correction for multiple comparisons. ${ }^{*} P$ $<0.05$, ${ }^{* *} P<0.01$, ${ }^{* *} P<0.001$, and ${ }^{* * * *} P<0.0001$ compared with the indicated group in the post hoc tests. in the CNS1 region of the Foxp3 gene (28). Moreover, SCFAs stimulate Treg proliferation through activation of GPR43 (29). Additional effects of SCFAs on Treg maturation are mediated by DCs (27). One report linked this effect to activation of the SCFA receptor GPR109A in DCs (50), while another did not confirm a role for GR109A and ascribed the activating effects of butyrate on DCs to HDAC inhibition (27). In line with these reports (27), in vitro studies revealed that butyrate was capable of increasing Treg differentiation by directly targeting T cells and DCs, while PTH was not. However, more physiologic ex vivo studies conducted by coculturing DCs and T cells harvested from mice treated in vivo with butyrate or iPTH revealed that the most relevant effect of butyrate was to increase the capacity of DCs to support Treg differentiation, whereas iPTH contributed to Treg differentiation by affecting $\mathrm{CD} 4^{+} \mathrm{T}$ cells through an indirect mechanism that remains to be determined. These findings were corroborated by data that showed that iPTH failed to induce bone anabolism and expand Tregs in GPR43/- mice. The expression of GPR43 is restricted to myeloid cells including DCs (28), confirming that PTH requires butyrate-induced DC activation to expand Tregs. On the other hand, maintenance of Tregs in unstimulated conditions was microbiota dependent but GPR43 independent, as GF and antibiotic-treated conventional mice had fewer intestinal and BM Tregs than microbiota-replete mice, while GPR $43^{-/-}$and WT littermates had similar number of Tregs.

Although depletion of the microbiota completely abrogated the bone anabolic activity of iPTH, conditional deletion of the PTH-PTHrP receptor in T cells blunts but does not abolish the anabolic activity of iPTH (17). These data further attest to the relevance of DCs as targets of butyrate and suggest that additional microbial products may mediate the effects of iPTH on targets other than $\mathrm{T}$ cells. Among the factors recognized to mediate the effects of iPTH on osteoblasts and to be microbial dependent is IGF-1 (51), which is also the first metabolite identi- 
A

$$
\text { - Veh • iPTH }
$$

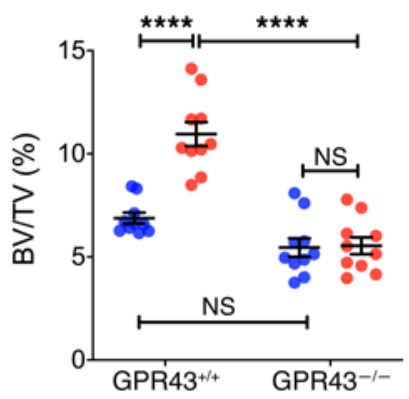

D

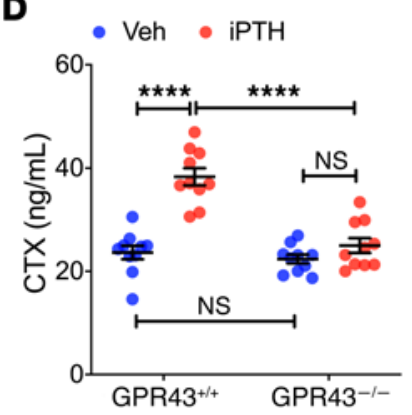

G

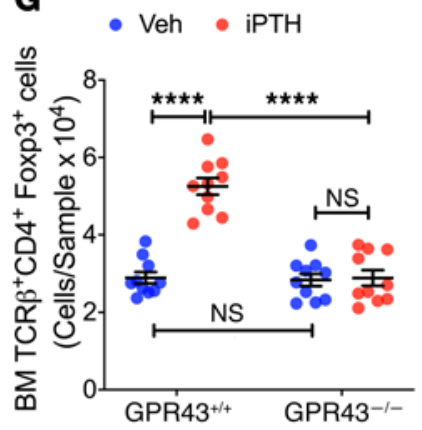

J

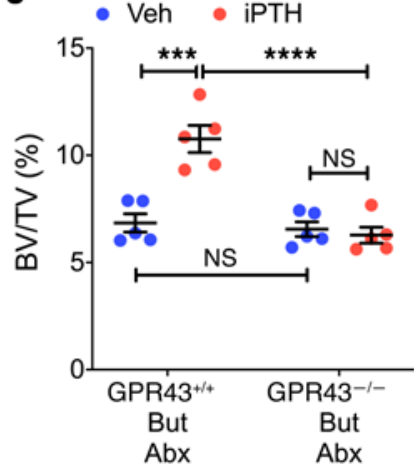

B

- Veh • IPTH

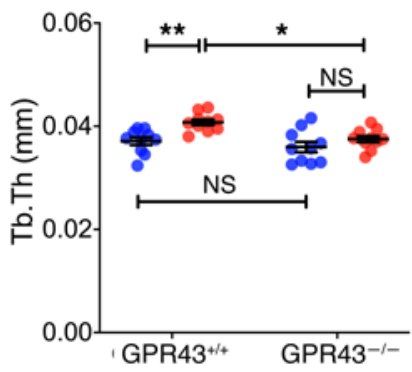

E

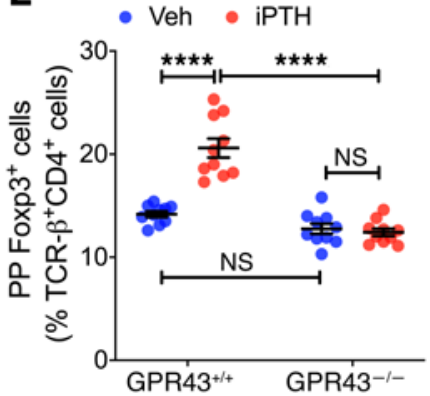

H

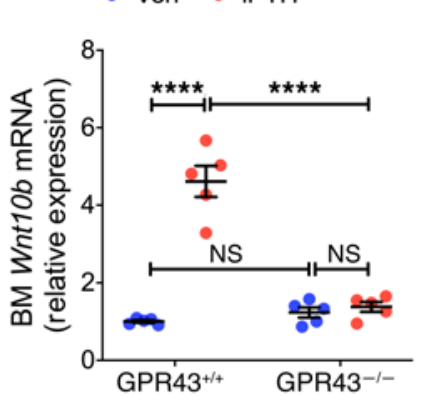

$\mathbf{K}$

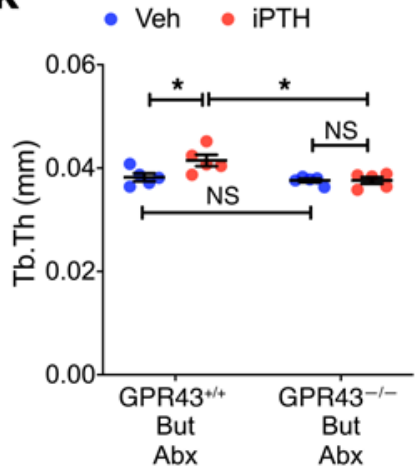

C

- Veh • iPTH

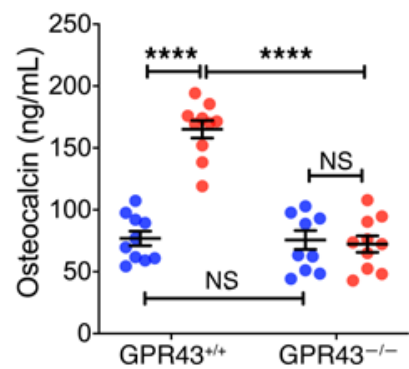

$\mathbf{F}$

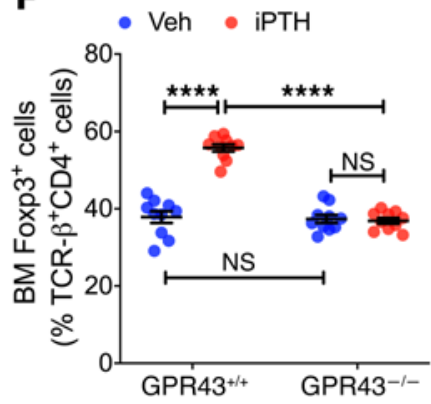

I

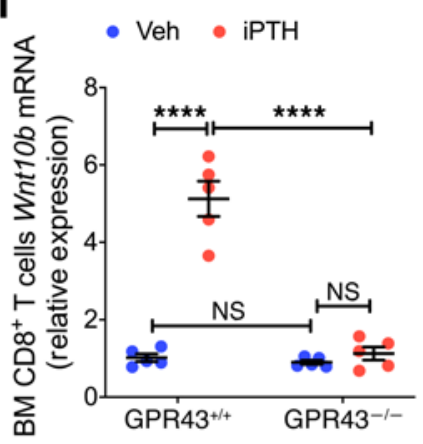

L

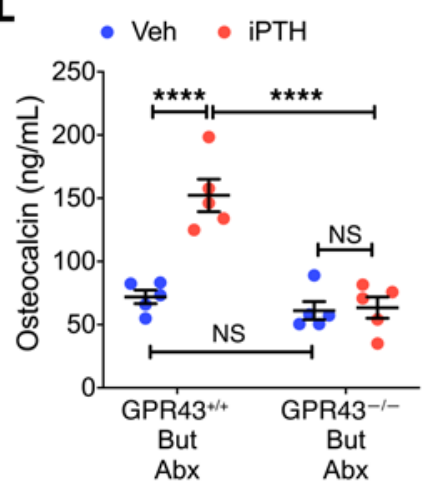

Figure 6. IPTH and butyrate fail to improve trabecular bone structure, stimulate bone turnover, expand Tregs, and induce Wn10b expression in 12-week-old GPR43--- mice. (A and B) $\mu C T$ scanning measurements of bone volume fraction (BV/TV) and trabecular thickness (Tb.Th) ( $n=10$ mice/group). (C and D) Serum osteocalcin and CTX levels ( $n=10$ mice/group). (E-C) PP and BM Tregs (TCR- $\beta^{+}$CD4+Foxp3+ cells) ( $n=10$ mice/group). (H and I) Wnt10b mRNA levels in whole BM cells and sorted BM CD8 ${ }^{+}$T cells $\left(n=5\right.$ mice/group). In A-I, GPR43-1- mice and WT littermates (CPR43 ${ }^{+/+}$mice) were treated with iPTH or vehicle for 4 weeks. Mice were sacrificed and analyzed at 12 weeks of age. (J-L) BV/TV, Tb.Th, and serum osteocalcin in mice treated with vehicle or iPTH, and butyrate (But) for 4 weeks starting at 8 weeks of age. Mice were also treated with antibiotics (Abx) for 6 weeks, starting at 6 weeks of age. Mice were sacrificed and analyzed at 12 weeks of age ( $n=5$ mice/group). Data were expressed as mean \pm SEM. All data were normally distributed according to the Shapiro-Wilk normality test. All data were analyzed by 2-way ANOVA and post hoc tests, applying Bonferroni's correction for multiple comparisons. ${ }^{*} P<0.05,{ }^{* *} P<0.01,{ }^{* *} P<0.001$, and ${ }^{* * * *} P<0.0001$ compared with the indicated group in the post hoc tests. 
fied as a link in the gut-bone axis $(31,52)$. Another bone-regulating molecule that could be permissive for the activity of iPTH is hydrogen sulfide, a gasotransmitter generated by bacteria residing within the gut $(53,54)$ that stimulates bone formation and postnatal skeletal development (55).

The bone histomorphometric analysis conducted in the current study revealed that ablation of the microbiota blocked not only the effects of iPTH on bone formation, but also those on trabecular bone resorption. It is unlikely that butyrate may enhance the proresorptive activity of $\mathrm{iPTH}$, as butyrate directly inhibits osteoclastogenesis and bone resorption (56). Also unlikely is the possibility that iPTH may stimulate bone resorption through $\mathrm{T}$ cell-produced Wnt10b, as silencing of Wnt10b expression in T cells does not block iPTH-induced bone resorption (16). By contrast, depletion of T cells blocks iPTH-induced bone resorption (16). Since iPTH decreases the expression of the anti-osteoclastogenic factor IFN- $\gamma$ by BM T cells (19), and considering that the microbiome regulates T cell expression of $\operatorname{IFN}-\gamma(57,58)$, it is likely that the microbiome is required for iPTH to blunt IFN- $\gamma$ production and thus stimulate bone resorption.

The bone anabolic activity of iPTH is, in part, mediated by $\mathrm{PTH}$ binding to the PTH/PTHrP receptor in osteocytes and the resulting blockade of the osteocytic production of the Wnt inhibitors sclerostin and Dkk1 (10-12). Importantly, deletion of PTH/ PTHrP in osteocytes blocks the bone anabolic activity of iPTH (13, 14) as effectively as depletion of the microbiota. This is consistent with a model in which independent and parallel activation of Wnt signaling by PTH binding to the PTH/PTHrP receptor in osteocytes and the microbiota/butyrate/Wnt10b pathway are both required for iPTH to stimulate bone formation.

The mechanism of action of iPTH described herein is surprisingly similar to the mechanism by which nutritional supplementation with the probiotic LGG stimulates bone formation and increase bone volume $(24,25)$. At bone anabolic doses, LGG increases the intestinal production of butyrate, causing an approximately 2 -fold increase in the circulating levels of butyrate. At this concentration, butyrate causes Treg expansion, Wnt10b secretion, and bone anabolism without the assistance of exogenous PTH. By contrast, physiologic concentrations of butyrate are required for iPTH to expand Tregs and induce bone anabolism.

Tregs stimulate $W n t 10 b$ gene expression in $\mathrm{CD}^{+} \mathrm{T}$ cells by silencing CD28 signaling, an event that lowers the production of AP- 1 by $\mathrm{CD}^{+} \mathrm{T}$ cells and shifts the associations of NFAT to SMADs rather than AP-1 (24). This increases the binding of NFAT/ SMAD dimers to a transcription-activating binding site located between $-705 \mathrm{bp}$ and $-272 \mathrm{bp}$ in the Wnt10b promoter (24). In mice treated with LGG, the recruitment of NFAT to the Wnt10b promoter is directly increased by butyrate. By contrast, in mice with physiologic levels of butyrate, it is likely that iPTH contributes to NFAT translocation by directly targeting $\mathrm{CD} 8^{+} \mathrm{T}$ cells, as PTH and PTHrP have previously been shown to directly activate NFAT signaling in other cell lineages $(59,60)$.

Osteoporosis is a common chronic disorder that represents a major source of disability in the elderly. An increase in the number of Tregs achievable by nutritional supplementation with butyrate may represent a novel therapeutic modality for osteoporosis or for potentiating the bone anabolic activity of PTH. Moreover, the use of butyrate to increase the number of Tregs may find wider applications, such as in transplant medicine or as a treatment for inflammatory and autoimmune conditions.

\section{Methods}

Animals. All in vivo experiments were carried out in female mice. Conventionally raised C57BL6 WT mice and C57BL6 TCR- $\beta^{-/-}$mice were acquired from Jackson Laboratory. C57BL6 GPR43-/- mice were supplied by Amgen. All mice were maintained under specific pathogen free conditions and fed sterilized food (5V5R chow) and autoclaved water ad libitum. Six-week-old female C57BL6 GF mice were purchased from Taconic Biosciences. Experimental GF mice were maintained in a Tecniplast ISOcage P-Bioexclusion System under a strict 12 hours light cycle and fed with autoclaved chow diet ad libitum within the Emory Gnotobiotic Animal Core (EGAC).

In vivo iPTH treatment. For the in vivo iPTH studies, $80 \mu \mathrm{g} / \mathrm{kg} / \mathrm{day}$ of hPTH 1-34 (Bachem California Inc.) or vehicle was injected daily subcutaneously into female mice for 4 weeks starting at the age of 8 weeks or at the age of 6 months, as described $(16-18,61)$.

Antibiotics and butyrate treatment. For antibiotic treatment, mice were given broad-spectrum antibiotics $(1 \mathrm{~g} / \mathrm{L}$ metronidazole, $1 \mathrm{~g} / \mathrm{L}$ ampicillin, $1 \mathrm{~g} / \mathrm{L}$ neomycin sulfate, and $0.5 \mathrm{~g} / \mathrm{L}$ vancomycin, MilliporeSigma) in drinking water. Depletion of microbiota was verified by quantitative real-time PCR (qPCR) using universal primer 16S rRNA. For butyrate administration, mice were treated with broad-spectrum antibiotics for 2 weeks, followed by supplementing $5 \mathrm{mM}$ butyrate in the antibiotic water for another 4 weeks. PTH or vehicle was injected into mice daily for 4 weeks starting at 2 weeks after the antibiotic treatment.

Excision of PPs from small intestine and preparation of single-cell suspension by mechanical dissociation. PP cell isolation was performed as described (62). Briefly, the small intestine was removed and flushed of fecal content. PPs were excised and collected in $1 \mathrm{~mL}$ cooled RPMI 1640. PPs were dissociated using the plunger of a $2.5 \mathrm{~mL}$ syringe and gently forced through a $70-\mu \mathrm{m}$ cell strainer placed over a $50 \mathrm{~mL}$ tube. A single cell suspension was used for analysis by flow cytometry.

$\mu \mathrm{CT}$ measurements. $\mu \mathrm{CT}$ scanning and analysis was performed using a Scanco $\mu$ CT-40 scanner, as previously reported $(18,63,64)$. Settings used for the in vitro scans are as follows: $70 \mathrm{kVp} 114 \mathrm{uA}$, $8 \mathrm{~W}$ with an integration time of $200 \mathrm{~ms}$ with a voxel size of $12 \mu \mathrm{m}^{3}$ for femurs from 12-week-old mice, and a voxel size of $10.5 \mu \mathrm{m}^{3}$ for femurs from 7-month-old mice. For the femoral trabecular region, we analyzed 70 slices, beginning 50 slices from the distal growth plate. Femoral cortical bone was measured using 80 continuous CT slides located at the femoral midshaft. We used the thresholding approach described by Bouxsein et al. (65), which is recommended by Scanco and includes a visual inspection and comparison of preview and slice-wise grayscale $2 \mathrm{D}$ images. The same threshold value was used for all measurements.

Quantitative bone histomorphometry. The measurements, terminology, and units used for histomorphometric analysis were those recommended by the Nomenclature Committee of the American Society of Bone and Mineral Research (66). Mice were injected subcutaneously with calcein at day 7 and day 2 before sacrifice. Nonconsecutive longitudinal sections of the femur ( $5-\mu \mathrm{m}$ thick) were cut from methyl methacrylate plastic-embedded blocks along the frontal plane using a Leica RM2155 microtome as described previously (63) and stained with Goldner's trichrome stain for the static measurements. Addi- 
A

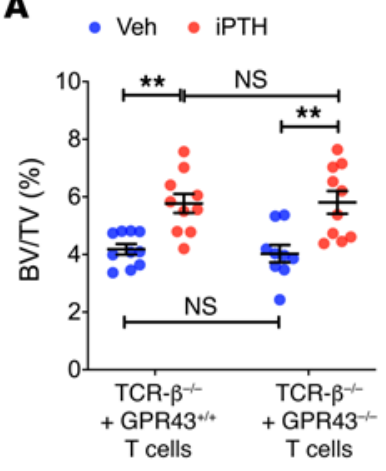

D

- Veh - iPTH

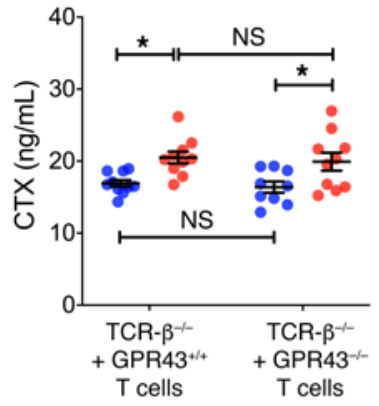

G

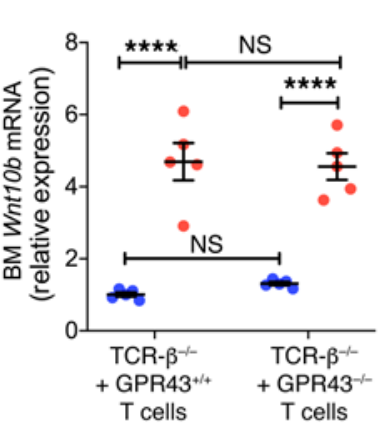

J

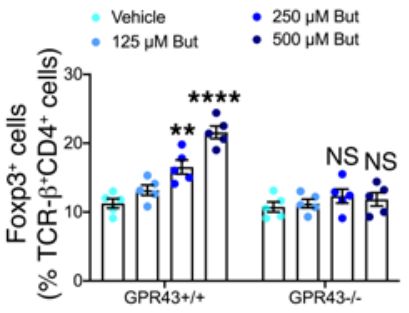

B

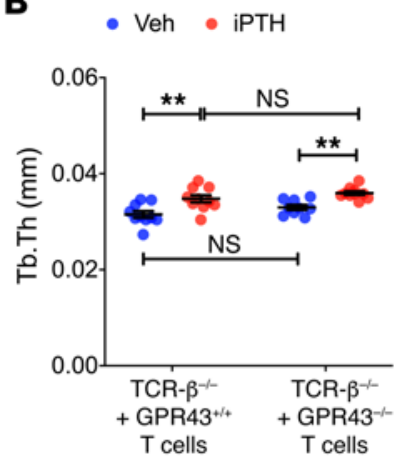

E
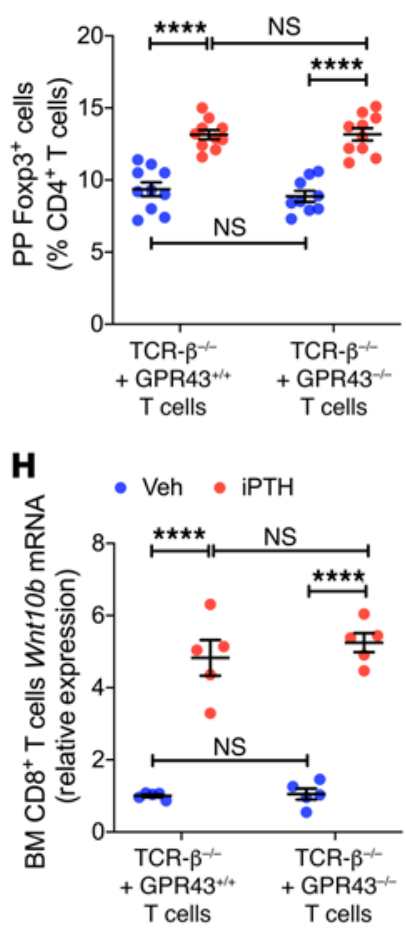

$\mathbf{K}$

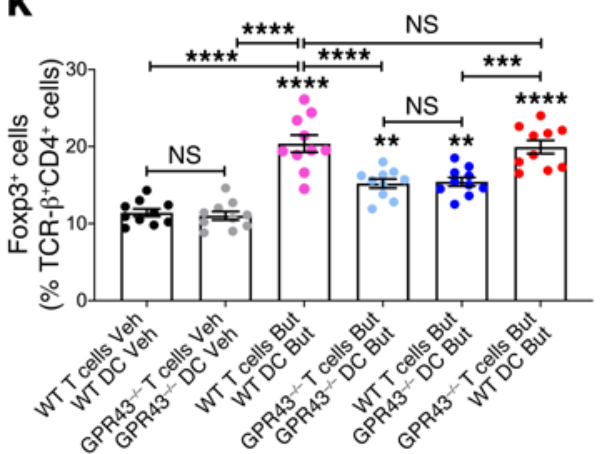

C

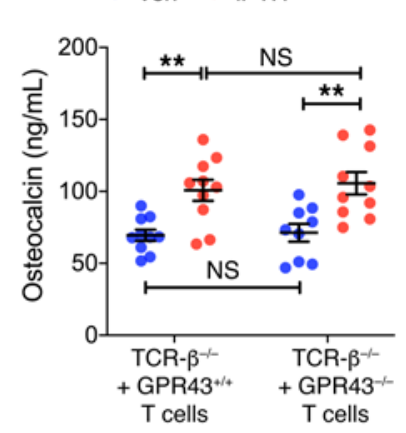

F $\bullet$ Veh $\bullet$ iPTH

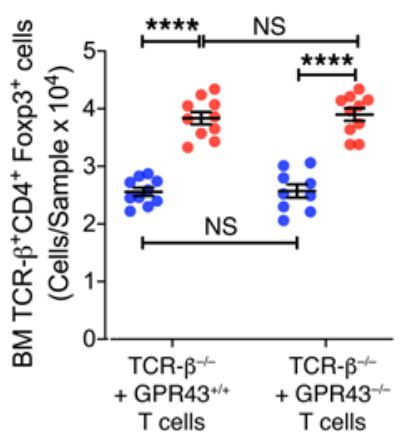

I

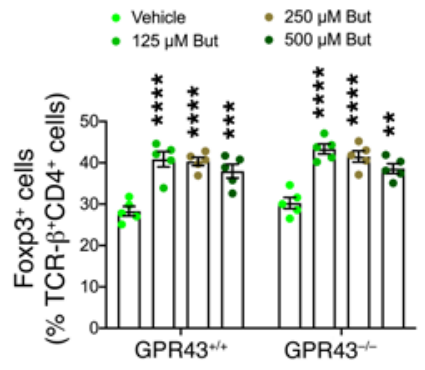

Figure 7. GPR43 signaling in DCs is required for IPTH treatment to improve trabecular structure, stimulate bone turnover, expand Tregs, and induce Wnt10b expression. TCR- $\beta^{-/-}$mice reconstituted with sorted splenic T cells from $\mathrm{GPR} 3^{-/-}$or GPR43+/+ littermates were treated with either vehicle or iPTH for 4 weeks. Mice were sacrificed and analyzed at 12 weeks of age. (A and $\mathbf{B}) \mu \mathrm{CT}$ scanning measurements of trabecular bone volume fraction (BV/TV) and trabecular thickness (Tb.Th) $(n=10$ mice/group). (C and D) Serum levels of osteocalcin and CTX ( $n=10$ mice/group). (E and F) PP and BM Tregs (CD4 ${ }^{+}$Foxp3 $^{+}$ cells) ( $n=10$ mice/group). (G and $\mathbf{H}$ ) Wnt10b mRNA levels in whole BM cells and sorted BM CD8 ${ }^{+} T$ cells ( $n=5$ mice) group). (I) Number of Tregs in cultures of naive $\mathrm{CD}^{+} \mathrm{T}$ cells from WT or GPR43-1in the presence of butyrate $(n=5 /$ group). (J) Number of Tregs in cocultures of WT naive CD4+ $T$ cells, and WT or CPR43 $3^{-1-}$ DCs pretreated with butyrate ( $n=5$ /group). (K) Number of Tregs in cocultures in naive $\mathrm{CD} 4^{+} \mathrm{T}$ cells and DCs. WT and GPR43-1- mice were treated with antibiotics for 4 weeks and iPTH or butyrate during the last 2 weeks $(n=10$ / group, from 2 separate experiments). In I, the cultures were stimulated by anti-CD3 and anti-CD28 antibodies, IL-2, and TGF- $\beta$. In J-K, the cultures were stimulated by anti-CD3 antibody and TGF- $\beta$. Data were expressed as mean \pm SEM. All data were normally distributed according to the Shapiro-Wilk normality test. Data in A-J were analyzed by 2-way ANOVA and post hoc tests, applying the Bonferroni's correction for multiple comparisons. Data in $\mathbf{K}$ were analyzed by 1-way ANOVA and post hoc tests, applying Bonferroni's correction for multiple comparisons ${ }^{*} P<0.05,{ }^{* *} P<$ $0.01,{ }^{* *} P<0.001$, and ${ }^{* * * *} P<0.0001$ compared with the indicated group in the post hoc tests.

tional sections were cut at $10 \mu \mathrm{m}$ and left unstained for dynamic (fluorescent) measurements. Trabecular measurements were obtained in an area of cancellous bone that measured approximately $2.5 \mathrm{~mm}^{2}$ and contained only secondary spongiosa, which was located 0.5-2.5 $\mathrm{mm}$ proximal to the epiphyseal growth cartilage of the femurs. Cortical measurements were obtained in a $1.5 \mathrm{~mm}$ area of the midshaft, which was located $4 \mathrm{~mm}$ below the epiphyseal growth cartilage. Measurements of single-labeled and double-labeled fluorescent surfaces and interlabel width were made in the same region of interest using unstained sections. MAR and BFR were calculated by the software by applying the interlabel period. Histomorphometry was done using the Bioquant Image Analysis System (R\&M Biometrics). 
Markers of bone turnover. Serum CTX and osteocalcin were measured by rodent-specific ELISA assays (Immunodiagnostic Systems).

Adoptive $T$ cell transfer. WT or GPR $43^{-/-}$spleen T cells purified by negative immunoselection using MACS Pan $\mathrm{T}$ cell isolation kit (Miltenyi Biotech) were injected $\left(5 \times 10^{6}\right.$ cells per mouse) IV into TCR- $\beta^{-/-}$recipient mice. T cells were transferred 2 weeks before initiating PTH injections to allow the engraftment and the peripheral expansion of the transferred T cells. Successful T cell engraftment was confirmed by flow cytometry of the spleens of the recipient mice harvested at sacrifice.

Stromal cells purification. BM SCs were purified as previously described (16-18). In brief, BM cells from long bones were cultured for 7 days in $\alpha$-MEM medium containing $10 \%$ FBS, $100 \mathrm{mg} / \mathrm{mL}$ penicillin, and $100 \mathrm{IU} / \mathrm{mL}$ streptomycin, to allow the proliferation of SCs. After removing nonadherent cells, adherent macrophages were eliminated by positive selection using anti-CD11c MACS Microbeads (Miltenyi Biotech). The remaining adherent cells were defined as SCs as they express alkaline phosphatase (ALP), type-I collagen, and Runx2, and have the capacity to form mineralization nodules when further cultured under mineralizing conditions.

SC thymidine incorporation assay. The proliferation of purified SCs was measured by $\left[{ }^{3} \mathrm{H}\right]$-thymidine incorporation assay. SCs were pulsed with $\left[{ }^{3} \mathrm{H}\right]$-thymidine $(0.5 \mu \mathrm{Ci} / 10,000$ cells $)$ for 18 hours, and were harvested with a Cell Harvestor (Skatron Inc.). [3H]-thymidine incorporation was read by a LS 6000 IC Liquid Scintillation Counter (Beckman Coulter Inc.).

SC apoptosis assay. The activity of caspase-3, the critical protease in the induction of apoptosis, was measured in SCs using CaspACE Assay System (Promega Corporation) according to the manufacturer's protocol.

Dendritic cell purification. BM DCs were isolated by MACS pos-

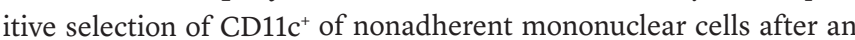
overnight adherence step to eliminate adherent cells. Cell purity was assessed by flow cytometry and found to be greater than $95 \%$.

In vitro Treg differentiation. Assessment of Treg differentiation in vitro was performed as described in the BioLegend protocol, "Treg Polarization of Mouse CD $4^{+}$Cells." Splenic CD $4^{+} \mathrm{T}$ cells from WT or GPR $43^{-/-}$mice were purified by using the EasySep Mouse Naive CD $4^{+}$ $\mathrm{T}$ Cell Isolation Kit (StemCell Technologies). To assess the direct effects of SCFA or PTH on T cells, naive $\mathrm{CD}^{+}{ }^{+} \mathrm{T}$ cells were cultured with titrated amounts of butyrate, propionate acetate, a mixture of these SCFAs, or PTH in the presence of anti-CD3 antibody (clone 145-2C11, BioLegend 100314) $(3 \mu \mathrm{g} / \mathrm{mL})$, anti-CD28 antibody (clone 37-51, BioLegend 102112) (3 $\mathrm{g} / \mathrm{mL}), \mathrm{IL}-2$ (2 ng/mL, BioLegend), and TGF- $\beta 1$ ( $1 \mathrm{ng} / \mathrm{mL}$, BioLegend) for 4 days. Cells were then harvested and analyzed by flow cytometry to enumerate $\mathrm{CD} 4^{+} \mathrm{Foxp} 3^{+}$cells. To assess the effects of butyrate or PTH on DCs, $2 \times 10^{4}$ BM DCs were cultured with titrated amounts of butyrate or PTH alone for 6 hours, washed, and cocultured with $1 \times 10^{5}$ naive $\mathrm{CD}^{+} \mathrm{T}$ cells in the presence of anti-CD3 antibody ( $3 \mu \mathrm{g} / \mathrm{mL})$ and TGF- $\beta 1(1 \mathrm{ng} / \mathrm{mL})$ in complete medium for 4 days. For ex vivo Tregs differentiation assays, $1 \times$ $10^{5}$ naive $\mathrm{CD} 4^{+} \mathrm{T}$ cells were cultured with $2 \times 10^{4} \mathrm{BM}$ DCs in 96-well round-bottom plates coated with anti-CD3 antibody $(3 \mu \mathrm{g} / \mathrm{mL})$ in the presence of TGF- $\beta 1(1 \mathrm{ng} / \mathrm{mL})$ in complete medium for 4 days. Naive $\mathrm{CD} 4^{+} \mathrm{T}$ cells and BM DCs were isolated from mice treated with broad-spectrum antibiotics for 4 weeks and butyrate or PTH during the last 2 weeks of the experiment.
Flow cytometry. Flow cytometry was performed on a LSR II system (BD Biosciences) and data were analyzed using FlowJo software (Tree Star Inc.). For intracellular Foxp3 staining, APC-Foxp3 (clone FJK16s, eBioscience, 17-5773-82) antibody was added after cell fixation and permeabilization with BD Transcription Factor Buffer Set (BD Biosciences). The following anti-mouse antibodies were used for cell surface staining: purified CD16/32 (clone 93, BioLegend, 101320), BV 421-TCR- $\beta$ (clone H57-597, Invitrogen, 48-5961-82), PerCP/Cy5.5CD4 (clone RM4-5, BioLegend, 100540), and BV 510-CD45 (clone 30-F11, BioLegend, 103137). The live cells were discriminated by Zombie NIR Fixable Viability Kit (BioLegend, 423105).

Butyrate quantification in serum samples. Measurement of butyrate levels was done by the Emory Integrated Lipidomics Core (EILC). SCFA extraction and derivation of serum samples were performed as described (24). Serum butyrate levels were measured by the internal standard method using the ExionLC AC system coupled to triple quadrupole mass spectrometer QTRAP5500 (AB Sciex). Peak determination, peak area integration, and calibration curves for standards were performed with the MultiQuant 3.0.2 software (AB Sciex).

Stool DNA extraction. Stool samples were collected directly into sterile tubes from live animals and snap-frozen before DNA extraction. Relative bacteria gene copies of GF mice and antibiotic-treated mice were confirmed at the end of the treatment period by fecal DNA extraction (DNA Stool Kit, Qiagen) as described in the manufacturer's protocol and subsequent q-PCR using 515F (GTGCCAGCMGCCGCGGTAA) and 806R (GGACTACHVGGGTWTCTAAT) primers. Conv.R mice and water were used as positive and negative controls, respectively.

Real-time RT-PCR and primers. RNA levels were quantified by qRT-PCR. RNA was isolated using the RNeasy kit (Qiagen) and cDNA was synthesized using Superscript II (Invitrogen) and random hexamers according to the manufacturer's instructions. Relative abundance of cDNAs was computed by quantitative RT-PCR analysis using the ABI StepOnePlus Real-Time PCR system (Applied Biosystems). $T g f b 1$ and $I g f 1 \mathrm{mRNA}$ levels were measured in gut and BM cells. Wnt10b mRNA levels were measured in BM cells, BM CD4 ${ }^{+} \mathrm{T}$ cells, and BM CD8 ${ }^{+} \mathrm{T}$ cells. The mRNA expression levels of bone sialoprotein $(B S P)$, collagen $1(C o L 1)$, osteocalcin $(O c n)$, osterix $(O s x)$, and runt-related transcription factor 2 (Runx2) were quantified in SCs. To investigate potential changes in Wnt-dependent gene expression, the mRNA levels of alyl-hydrocarbon receptor (Ahr), axin2 (Axin2), cystein rich protein 61 (Cyr61), transgelin (Tagln), transforming growth factor beta 3 (Tgfb3), thrombospondin 1 (Thbs1), twist gene homolog 1(Twist1), and Wnt1 inducible signaling pathway protein 1 (Wisp1) were assessed in SC. All the primers used were designed by Primer Express Ò Software v3.0.1 (Applied Biosystems). Changes in relative gene expression between vehicle and Leuprolide groups were calculated using the $2^{-\Delta \Delta C T}$ method with normalization to $18 \mathrm{~S}$ rRNA. The primers used were as follows: $5^{\prime}$-ATTCGAACGTCTGCCCTATCA-3' (forward) and 5'-GTCACCCGTGGTCACCATG-3' (reverse) for 18s rRNA, 5'-CTCTCCGACCTGCCACAGA-3' (forward) and 5'-CCAATAAAGGCACCCTATGACTCT-3' (reverse) for Tgfb1, 5'-AGCTGGTGGATGCTCTTCGTT-3' (forward) and 5'-ATCCACAATCCCTGTCTGGT-3' (reverse) for Igf1, 5'-GGGACCTCGGGTGACAATAA-3' (forward) and 5'-CCTCTGTCCTTTTCCAACCG-3' (reverse) for Wnt10b, 5'-GGGACCTCGGGTGACAATAA-3' (forward) and 5'-CCTCTGTCCTTTTCCAACCG-3' (reverse) for Ahr, 5'-CAGTGTGAAGGCCAATGGC-3' (forward) and 5'-TGG- 
GTTCTCGGAAAATGAGG-3' (reverse) for Axin-2, 5'-GTGAAGTGCGTCCTTGTGGA-3' (forward) and 5'-TGCCCTTTTTTAGGCTGCTG-3' (reverse) for Cyr61, 5'-AATTTCAGTCCAAGCACGCC-3' (forward) and 5'-CGGGACTCTCTCTCCTCTTGC-3' (reverse) for $\quad N k d 2, \quad 5^{\prime}$-CAGCCCAGACACCGAAGCTA-3' (forward) and 5'-AgGCTTGGTCGTTTGTGGAC-3' (reverse) for Tagln, 5'-GCAAAGGGCTCTGGTAGTCCT-3' (forward) and 5'-AGGCTGATTGTGGCCAAGTT-3' (reverse) for Tgfb3, 5'-GGACCGGGCTCAACTCTACA-3' (forward) and 5'-AGCTCCGCGCTCTCCAT-3' (reverse) for Thbs1, 5'-TCGACTTCCTGTACCAGGTCCT-3' (forward) and 5'-CCATCTTGGAGTCCAGCTCG-3' (reverse) for Twist1, and 5'-ATGCCTGGCTGTGTACCAGC-3' (forward) and 5'-CCTGCGAGAGTGAAGTTCGTG-3' (reverse) for Wsp1.

Statistics. All data are expressed as mean \pm SEM. When data were normally distributed according to the Shapiro-Wilk normality test, data were analyzed by unpaired $t$ tests, 1-way ANOVA, or 2-way ANOVA as appropriate. This latter analysis included the main effects for animal strain and treatment plus the statistical interaction between animal strain and treatment. When the statistical interaction was statistically significant $(P<0.05)$ or suggestive of an important interaction, then $t$ tests were used to compare the differences between the treatment means for each animal strain, applying the Bonferroni's correction for multiple comparisons. Data that were not normally distributed (as tested by Shapiro-Wilk normality test) were analyzed by Kruskal-Wallis nonparametric tests and Dunn's multiple comparisons nonparametric tests.
Study approval. All the animal procedures were approved by the Institutional Animal Care and Use Committee of Emory University.

\section{Author contributions}

JYL, MNW, RMJ, and RP designed the studies. JYL, MY, AMT, SP, $\mathrm{HD}$, and JA performed the research and analyzed the animal data. $\mathrm{RP}, \mathrm{MNW}$, and RMJ wrote the manuscript.

\section{Acknowledgments}

This study was supported by grants from the National Institutes of Health (DK112946, DK108842, and RR028009 to RP; AG062334, AR068157, and AR070091 to MNW; DK098391 to RMJ). MNW was also supported by a grant from the Biomedical Laboratory Research \& Development Service of the VA Office of Research and Development (5I01BX000105). EGAC is supported by the Georgia Clinical \& Translational Science Alliance and the Emory University School of Medicine. ELIC is supported by the Emory Neuroscience NINDS Core Facilities, the Georgia Clinical \& Translational Science Alliance, and the Emory University School of Medicine.

Address correspondence to: Roberto Pacifici, Division of Endocrinology, Metabolism and Lipids, Emory University School of Medicine, 101 Woodruff Circle, Room 1307, Atlanta, Georgia 30322, USA. Phone: 404.712.8420; Email: roberto.pacifici@emory.edu.
1. Wein MN, Kronenberg HM. Regulation of bone remodeling by parathyroid hormone. Cold Spring Harb Perspect Med. 2018;8(8):a031237.

2. Miao D, He B, Karaplis AC, Goltzman D. Parathyroid hormone is essential for normal fetal bone formation. JClin Invest. 2002;109(9):1173-1182.

3. Iida-Klein A, et al. Short-term continuous infusion of human parathyroid hormone 1-34 fragment is catabolic with decreased trabecular connectivity density accompanied by hypercalcemia in C57BL/J6 mice. JEndocrinol. 2005;186(3):549-557.

4. Bilezikian JP. Primary hyperparathyroidism. JClin Endocrinol Metab. 2018;103(11):3993-4004.

5. Potts J. Chapter 13. In: Avioli LV, Krane S, eds. Metabolic Bone Diseases. San Diego, CA: Academic Press; 1998:411-442.

6. Uzawa T, Hori M, Ejiri S, Ozawa H. Comparison of the effects of intermittent and continuous administration of human parathyroid hormone(1-34) on rat bone. Bone. 1995;16(4):477-484.

7. Jilka RL. Molecular and cellular mechanisms of the anabolic effect of intermittent PTH. Bone. 2007;40(6):1434-1446.

8. Qin L, Raggatt LJ, Partridge NC. Parathyroid hormone: a double-edged sword for bone metabolism. Trends Endocrinol Metab. 2004;15(2):60-65.

9. Keller H, Kneissel M. SOST is a target gene for PTH in bone. Bone. 2005;37(2):148-158.

10. Bellido T, et al. Chronic elevation of parathyroid hormone in mice reduces expression of sclerostin by osteocytes: a novel mechanism for hormonal control of osteoblastogenesis. Endocrinology. 2005;146(11):4577-4583.

11. Silvestrini G, et al. Effects of intermittent parathyroid hormone (PTH) administration on SOST
mRNA and protein in rat bone. J Mol Histol. 2007;38(4):261-269.

12. Guo J, et al. Suppression of Wnt signaling by Dkk1 attenuates PTH-mediated stromal cell response and new bone formation. Cell Metab. 2010;11(2):161-171.

13. Saini V, et al. Parathyroid hormone (PTH)/ PTH-related peptide type 1 receptor (PPR) signaling in osteocytes regulates anabolic and catabolic skeletal responses to PTH. JBiol Chem. 2013;288(28):20122-20134.

14. Delgado-Calle J, et al. Control of bone anabolism in response to mechanical loading and PTH by distinct mechanisms downstream of the PTH receptor. J Bone Miner Res. 2017;32(3):522-535.

15. D'Amelio P, et al. Treatment with intermittent PTH increases Wnt10b production by $\mathrm{T}$ cells in osteoporotic patients. Osteoporos Int. 2015;26(12):2785-2791.

16. Terauchi M, et al. T lymphocytes amplify the anabolic activity of parathyroid hormone through Wnt1Ob signaling. Cell Metab. 2009;10(3):229-240.

17. Bedi B, et al. Silencing of parathyroid hormone (PTH) receptor 1 in T cells blunts the bone anabolic activity of PTH. Proc Natl Acad Sci US A. 2012;109(12):E725-E733.

18. Li JY, Walker LD, Tyagi AM, Adams J, Weitzmann MN, Pacifici R. The sclerostin-independent bone anabolic activity of intermittent PTH treatment is mediated by T-cell-produced Wnt10b. J Bone Miner Res. 2014;29(1):43-54.

19. Yu M, et al. Regulatory T cells are expanded by teriparatide treatment in humans and mediate intermittent PTH-induced bone anabolism in mice. EMBO Rep. 2018;19(1):156-171.
20. Shevach EM. Mechanisms of foxp3+ T regulatory cell-mediated suppression. Immunity. 2009;30(5):636-645.

21. Kim YG, Lee CK, Nah SS, Mun SH, Yoo B, Moon HB. Human CD4+CD25+ regulatory T cells inhibit the differentiation of osteoclasts from peripheral blood mononuclear cells. Biochem Biophys Res Commun. 2007;357(4):1046-1052.

22. Kelchtermans H, Geboes L, Mitera T, Huskens D, Leclercq G, Matthys P. Activated CD4+CD25+ regulatory $\mathrm{T}$ cells inhibit osteoclastogenesis and collagen-induced arthritis. Ann Rheum Dis. 2009;68(5):744-750.

23. Lei H, Schmidt-Bleek K, Dienelt A, Reinke P, Volk HD. Regulatory T cell-mediated anti-inflammatory effects promote successful tissue repair in both indirect and direct manners. Front Pharmacol. 2015;6:184.

24. Tyagi AM, et al. The microbial metabolite butyrate stimulates bone formation via T regulatory cell-mediated regulation of WNT10B expression. Immunity. 2018;49(6):1116-1131.e7.

25. Zaiss MM, Jones RM, Schett G, Pacifici R. The gut-bone axis: how bacterial metabolites bridge the distance. J Clin Invest. 2019;129(8):3018-3028.

26. Bach Knudsen KE. Microbial degradation of whole-grain complex carbohydrates and impact on short-chain fatty acids and health. Adv Nutr. 2015;6(2):206-213.

27. Arpaia N, et al. Metabolites produced by commensal bacteria promote peripheral regulatory T-cell generation. Nature. 2013;504(7480):451-455.

28. Furusawa Y, et al. Commensal microbe-derived butyrate induces the differentiation of colonic regulatory T cells. Nature. 2013;504(7480):446-450. 
29. Smith PM, et al. The microbial metabolites, short-chain fatty acids, regulate colonic Treg cell homeostasis. Science. 2013;341(6145):569-573.

30. Sjögren $\mathrm{K}$, et al. The gut microbiota regulates bone mass in mice. JBone Miner Res. 2012;27(6):1357-1367.

31. Novince CM, et al. Commensal gut microbiota immunomodulatory actions in bone marrow and liver have catabolic effects on skeletal homeostasis in health. Sci Rep. 2017;7(1):5747.

32. Jackson A, et al. Gene array analysis of Wntregulated genes in C3H10T1/ 2 cells. Bone. 2005;36(4):585-598.

33. Tanoue T, Atarashi K, Honda K. Development and maintenance of intestinal regulatory $\mathrm{T}$ cells. Nat Rev Immunol. 2016;16(5):295-309.

34. Anguela XM, et al. Nonviral-mediated hepatic expression of IGF-I increases Treg levels and suppresses autoimmune diabetes in mice. Diabetes. 2013;62(2):551-560.

35. Johannesson $\mathrm{B}$, et al. Insulin-like growth factor-1 induces regulatory $\mathrm{T}$ cell-mediated suppression of allergic contact dermatitis in mice. Dis Model Mech. 2014;7(8):977-985.

36. Oursler MJ, et al. Modulation of transforming growth factor-beta production in normal human osteoblast-like cells by 17 betaestradiol and parathyroid hormone. Endocrinology. 1991;129(6):3313-3320.

37. Wu Y, Kumar R. Parathyroid hormone regulates transforming growth factor beta1 and beta2 synthesis in osteoblasts via divergent signaling pathways. J Bone Miner Res. 2000;15(5):879-884.

38. McCarthy TL, Centrella M, Canalis E. Parathyroid hormone enhances the transcript and polypeptide levels of insulin-like growth factor I in osteoblast-enriched cultures from fetal rat bone. Endocrinology. 1989;124(3):1247-1253.

39. Annison G, Illman RJ, Topping DL. Acetylated, propionylated or butyrylated starches raise large bowel short-chain fatty acids preferentially when fed to rats. J Nutr. 2003;133(11):3523-3528.

40. Nilsson NE, Kotarsky K, Owman C, Olde B. Identification of a free fatty acid receptor, FFA2R, expressed on leukocytes and activated by short-chain fatty acids. Biochem Biophys Res Commun. 2003;303(4):1047-1052.

41. Maslowski KM, et al. Regulation of inflam- matory responses by gut microbiota and chemoattractant receptor GPR43. Nature. 2009;461(7268):1282-1286.

42. Fujisaki J, et al. In vivo imaging of Treg cells providing immune privilege to the haematopoietic stem-cell niche. Nature. 2011;474(7350):216-219.

43. Zaiss MM, et al. Treg cells suppress osteoclast formation: a new link between the immune system and bone. Arthritis Rheum. 2007;56(12):4104-4112.

44. Yuan FL, et al. Regulatory T cells as a potent target for controlling bone loss. Biochem Biophys Res Commun. 2010;402(2):173-176.

45. Zaiss MM, et al. Increased bone density and resistance to ovariectomy-induced bone loss in FoxP3-transgenic mice based on impaired osteoclast differentiation. Arthritis Rheum. 2010;62(8):2328-2338.

46. Banchereau J, Steinman RM. Dendritic cells and the control of immunity. Nature. 1998;392(6673):245-252.

47. Cario E. Bacterial interactions with cells of the intestinal mucosa: Toll-like receptors and NOD2. Gut. 2005;54(8):1182-1193.

48. Setoguchi R, Hori S, Takahashi T, Sakaguchi S. Homeostatic maintenance of natural Foxp3(+) CD25(+) CD4(+) regulatory T cells by interleukin (IL)-2 and induction of autoimmune disease by IL-2 neutralization. JExp Med. 2005;201(5):723-735.

49. Zeng H, Chi H. Metabolic control of regulatory $\mathrm{T}$ cell development and function. Trends Immunol. 2015;36(1):3-12.

50. Singh N, et al. Activation of Gpr109a, receptor for niacin and the commensal metabolite butyrate, suppresses colonic inflammation and carcinogenesis. Immunity. 2014;40(1):128-139.

51. Bikle DD, et al. Insulin-like growth factor I is required for the anabolic actions of parathyroid hormone on mouse bone. J Bone Miner Res. 2002;17(9):1570-1578.

52. Yan J, et al. Gut microbiota induce IGF-1 and promote bone formation and growth. Proc Natl Acad Sci U S A. 2016;113(47):E7554-E7563.

53. Linden DR. Hydrogen sulfide signaling in the gastrointestinal tract. Antioxid Redox Signal. 2014;20(5):818-830.

54. Shen X, Carlström M, Borniquel S, Jädert C, Kevil CG, Lundberg JO. Microbial regulation of host hydrogen sulfide bioavailability and metabolism.
Free Radic Biol Med. 2013;60:195-200.

55. Liu Y, et al. Hydrogen sulfide maintains mesenchymal stem cell function and bone homeostasis via regulation of $\mathrm{Ca}(2+)$ channel sulfhydration. Cell Stem Cell. 2014;15(1):66-78.

56 . Lucas $S$, et al. Short-chain fatty acids regulate systemic bone mass and protect from pathological bone loss. Nat Commun. 2018;9(1):55.

57. Imanishi T, Hara H, Suzuki S, Suzuki N, Akira S, Saito T. Cutting edge: TLR2 directly triggers Th1 effector functions. JImmunol. 2007;178(11):6715-6719.

58 . Ye L, Schnepf D, Staeheli P. Interferon- $\lambda$ orchestrates innate and adaptive mucosal immune responses. Nat Rev Immunol. 2019;19(10):614-625.

59. Park HJ, Baek K, Baek JH, Kim HR. The cooperation of CREB and NFAT is required for PTHrP-induced RANKL expression in mouse osteoblastic cells. J Cell Physiol. 2015;230(3):667-679.

60. Huang $\mathrm{H}$, et al. Parathyroid hormone induction of cyclooxygenase-2 in murine osteoblasts: role of the calcium-calcineurin-NFAT pathway. J Bone Miner Res. 2010;25(4):819-829.

61. Li JY, et al. PTH expands short-term murine hemopoietic stem cells through T cells. Blood. 2012;120(22):4352-4362.

62. Lefrançois L, Lycke N. Isolation of mouse small intestinal intraepithelial lymphocytes, Peyer's patch, and lamina propria cells. Curr Protoc Immunol. 2001; Chapter 3:Unit 3.19.

63. Li JY, et al. IL-17A Is increased in humans with primary hyperparathyroidism and mediates PTH-induced bone loss in mice. Cell Metab. 2015;22(5):799-810.

64. Robinson JW, et al. T cell-expressed CD4OL potentiates the bone anabolic activity of intermittent PTH treatment. J Bone Miner Res. 2015;30(4):695-705

65. Bouxsein ML, Boyd SK, Christiansen BA, Guldberg RE, Jepsen KJ, Müller R. Guidelines for assessment of bone microstructure in rodents using micro-computed tomography. J Bone Miner Res. 2010;25(7):1468-1486.

66. Dempster DW, et al. Standardized nomenclature, symbols, and units for bone histomorphometry: a 2012 update of the report of the ASBMR Histomorphometry Nomenclature Committee. J Bone Miner Res. 2013;28(1):2-17. 